\title{
Time-course differential IncRNA and mRNA expressions in radioresistant hypopharyngeal cancer cells
}

\author{
Jieyu Zhou ${ }^{1,2, *}$, Shengda $\mathrm{Cao}^{1, *}$, Wenming $\mathrm{Li}^{1}$, Dongmin Wei ${ }^{1}$, Zhentao Wang ${ }^{2}$, \\ Guojun Li ${ }^{3,4}$, Xinliang Pan $^{1}$ and Dapeng Lei ${ }^{1}$ \\ ${ }^{1}$ Department of Otorhinolaryngology, Qilu Hospital, Shandong University, Key Laboratory of Otolaryngology, NHFPC - \\ Shandong University, Jinan, Shandong, 250012, P.R. China \\ ${ }^{2}$ Department of Otorhinolaryngology, Shanghai Ninth People's Hospital, Shanghai Jiaotong University, School of Medicine, \\ Shanghai, 200011, P.R. China \\ ${ }^{3}$ Department of Head and Neck Surgery, The University of Texas MD Anderson Cancer Center, Houston, TX, 77030, USA \\ ${ }^{4}$ Department of Epidemiology, The University of Texas MD Anderson Cancer Center, Houston, TX, 77030, USA \\ *These authors contributed equally to this work \\ Correspondence to: Xinliang Pan, email: panxin1915@126.com \\ Dapeng Lei, email: leidapeng@sdu.edu.cn \\ Keywords: hypopharyngeal squamous cell carcinoma, radioresistance, IncRNA, mRNA, microarray \\ Received: December 05, $2016 \quad$ Accepted: April 10, $2017 \quad$ Published: April 21, 2017 \\ Copyright: Zhou et al. This is an open-access article distributed under the terms of the Creative Commons Attribution License 3.0 (CC BY \\ $3.0)$, which permits unrestricted use, distribution, and reproduction in any medium, provided the original author and source are credited.
}

\section{ABSTRACT}

Radioresistance remains a major problem in the treatment of patients with hypopharyngeal squamous cell carcinoma (HSCC). Long noncoding RNAs (IncRNAs) have important roles in the development, invasion, and metastasis of various tumors, including HSCC, but little is known about the role of IncRNAs in cancer radioresistance. The aim of this study was to identify radioresistance-related IncRNAs and mRNAs in radioresistant (RS) hypopharyngeal cancer subclone RS-FaDu cells. In this study, we performed microarray analysis to find the differences in timecourse IncRNA and mRNA expression profiles between RS-FaDu and parent FaDu cells after $4 \mathrm{~Gy}$ radiation therapy, whose reliability was confirmed by validation experiment. Among these consistently dysregulated IncRNAs, we found that some IncRNAs (e.g., TCONS_00018436) might control resistance of HSCC cells to radiation. Furthermore, our bioinformatics analyses from mRNA/IncRNA microarray data showed that certain IncRNAs or mRNAs potentially are involved in radioresistance of HSCC. Our results from this study laid the foundation for further investigating the roles of these IncRNAs and mRNAs as promising candidates in the occurrence and development of HSCC radioresistance.

\section{INTRODUCTION}

Hypopharyngeal squamous cell carcinoma (HSCC), which originates from the mucosa of the hypopharynx, has one of the poorest prognoses among head and neck cancers [1]. Currently, the standard treatment strategy for HSCC is surgery followed by radiotherapy [1]. Significant advances in radiotherapeutic strategies for HSCC, such as intensity-modulated radiotherapy (IMRT), image-guided radiotherapy (IGRT), and helical tomotherapy (TOMO), have been made in recent years $[1,2]$. However, local recurrence and distant metastases after radiotherapy due to tumor radioresistance remain a serious obstacle to successful treatment of HSCC, and the 5-year survival rate remains at approximately $25 \%$ to $40 \%$ [3]. Although mechanisms of radioresistance have been extensively studied $[4,5]$, the underlying molecular pathways and targets involved in HSCC radioresistance are not fully understood. Currently, there are few strategies available for overcoming this clinical problem.

In the past decade, advances in genome-wide analysis of gene expression have revealed that the majority of genes in the genome are transcribed into non-coding RNAs (ncRNAs) [6]. Long non-coding RNAs (lncRNAs) are ncRNAs longer than 200 nucleotides [7], and they have important roles in chromatin modification and 
transcriptional and post-transcriptional processing [8, 9]. Specifically, lncRNAs have been demonstrated to promote the development, invasion, and metastasis of many tumors by a variety of mechanisms [10, 11]. Notably, several studies have shown that lncRNAs are extremely important for controlling cancer radioresistance [12-15], but the roles of lncRNAs in HSCC radioresistance are still unknown.

Accumulating evidence demonstrates that lncRNAs are widely involved in the regulation of proliferation, DNA damage response, apoptosis, and the cell cycle in cancer cells [16-22], all of which are closely associated with the development of radioresistance [23]. Moreover, the roles of several lncRNAs, such as MALAT1 [13, 24], TUG1 [14], NEAT1 [25], and BOKAS [26], in radioresistance have been identified, although their detailed mechanisms remain largely unclear. Radioresistance is the leading cause of recurrence and poor prognosis in HSCC patients. Hence, it is of vital significance to figure out whether or not lncRNAs can become biomarkers for radioresistant HSCC and explore the molecular mechanisms underlying HSCC radioresistance.

It is very unlikely that a single molecule or gene is responsible for radioresistance in HSCC; therefore, to provide useful information for elucidating the molecular mechanisms that lncRNAs and mRNAs are involved in HSCC radioresistance, we used microarray techniques to perform large-scale analyses of lncRNA and mRNA expressions to comprehensively search for mechanisms of HSCC radioresistance. We initially generated a radioresistant HSCC subclone ( $\mathrm{RS}-\mathrm{FaDu}$ ) from the parental $\mathrm{FaDu}$ cell line via longterm fractionated irradiation. Subsequently, we investigated differences in time-course lncRNA and mRNA expression profiles between RS-FaDu and parent FaDu cells after radiation therapy by microarray and bioinformatics analyses.

\section{RESULTS}

\section{Establishment and validation of radioresistant HSCC subclone cell line}

Radioresistance was measured by clonogenic survival assay following exposure to a range of radiation doses (0-6 Gy). As shown in Figure 1A, RS-FaDu and $\mathrm{FaDu}$ cells showed no difference in clonogenic formation ability when the radiation dose was 0 Gy. However, the RS-FaDu cells had significantly more and larger surviving colonies than did the control FaDu cells when the radiation dose was 4 Gy or 6 Gy. Clonogenic survival curves showed the surviving colony numbers of $\mathrm{FaDu}$ cells were significantly lower than those of RS-FaDu cells $(* * P<0.01$, both) at 4 Gy and 6 Gy (Figure 1B).

To further verify the radioresistant phenotype of RS$\mathrm{FaDu}, \mathrm{RS}-\mathrm{FaDu}$ and $\mathrm{FaDu}$ cells were also examined by apoptosis assays. RS-FaDu and $\mathrm{FaDu}$ cells were treated with 4 Gy. Their fractions of apoptosis cells did not differ at $0 \mathrm{~h}$, but at 24,48 , or $72 \mathrm{~h}$ after irradiation, the fractions of apoptotic RS-FaDu cells were much lower than those of FaDu cells $(* * P<0.01$, Figure $1 \mathrm{C}, 1 \mathrm{D})$. These results indicated that RS-FaDu cells were much more radioresistant than their parent FaDu cells. These data indicated that the RS-FaDu subclone cell line was successfully established.

\section{LncRNA and mRNA profiles}

Hierarchical clustering is an unsupervised classification method that can separate multiple groups without the use of the group information. In microarray data analysis, cluster analysis grouped samples together based on expression intensity revealed differences between clustering group and true group results for removal of outlier samples. The dendrogram showed the relationships among lncRNA (Figure 2A) and mRNA (Figure 2B) expression patterns between $\mathrm{RS}-\mathrm{FaDu}$ cells and $\mathrm{FaDu}$ cells at 0,2 , and $48 \mathrm{~h}$, respectively, after 4 Gy radiation exposure.

A scatter plot is a visualization method to show the differentially expressed lncRNAs (Figure 3, A1-C1) and mRNAs (Figure 3, D1-F1). The values plotted on the $\mathrm{X}$ and $\mathrm{Y}$ axes were the averaged normalized signal values of groups of samples ( $\log 2$ scaled). The $\mathrm{X}$-axis represents the control group $(\mathrm{FaDu})$, while the $\mathrm{Y}$-axis represents the case group (RS-FaDu). All lncRNAs/mRNAs that were not differentially expressed were around the line $\mathrm{Y}=\mathrm{X}$ and labelled black. Points that were above and apart from $\mathrm{Y}=\mathrm{X}$ were upregulated $\operatorname{lncRNAs} / \mathrm{mRNAs}$ and labelled red, and points that were below and apart from $\mathrm{Y}=\mathrm{X}$ were downregulated lncRNAs/mRNAs and labelled green, respectively. A volcano plot is another visualization method to show differences in lncRNA (Figure 3, A2-C2) and mRNA (Figure 3, D2-F2) expression between the case group ( $\mathrm{RS}-\mathrm{FaDu}$ ) and the control group (FaDu). The X-axis in the volcano plot represents FC (after log2 transformation) and the Y-axis represents $P$-value (after $\log$ transformation). The lncRNAs/mRNAs on the top left were downregulated $\operatorname{lncRNAs} / \mathrm{mRNAs}$ (FC $\geq 2.0$, $P$ value $<0.05)$. The $\operatorname{lncRNAs} / \mathrm{mRNAs}$ on the top right were upregulated $\operatorname{lncRNAs} / \mathrm{mRNAs}$ (FC $\geq 2.0$, $P$ value $<0.05)$. Upregulated 1 chNAs/mRNAs and downregulated lncRNAs/mRNAs were labelled red and green, respectively. After the RS-FaDu and FaDu cells were treated with 4 Gy of irradiation, their lncRNA expression levels differed significantly in 575, 361, and 1714 lncRNAs (data not shown) at 0,2 , and $48 \mathrm{~h}$, respectively. Of those, 302 were upregulated and 273 were downregulated at $0 \mathrm{~h} ; 113$ were upregulated and 248 were downregulated at $2 \mathrm{~h}$; and 759 were upregulated and 955 were downregulated at $48 \mathrm{~h}$, respectively. In addition, we identified 1249, 781, and 2521 (data not shown) mRNAs that were significantly differentially expressed at 0,2 , and 48 h, respectively. Of those, 387 were upregulated and 862 were downregulated at $0 \mathrm{~h} ; 227$ were upregulated and 554 were downregulated at $2 \mathrm{~h}$; and 1089 were upregulated and 1432 were downregulated at $48 \mathrm{~h}$. Volcano plot filtering was also used to identify the 10 most upregulated and 


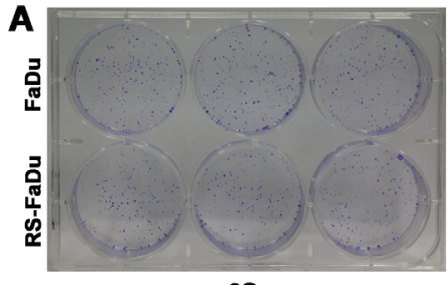

OGy

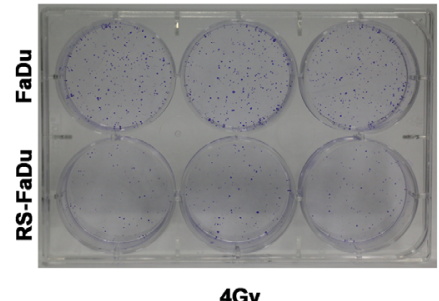

4Gy

C

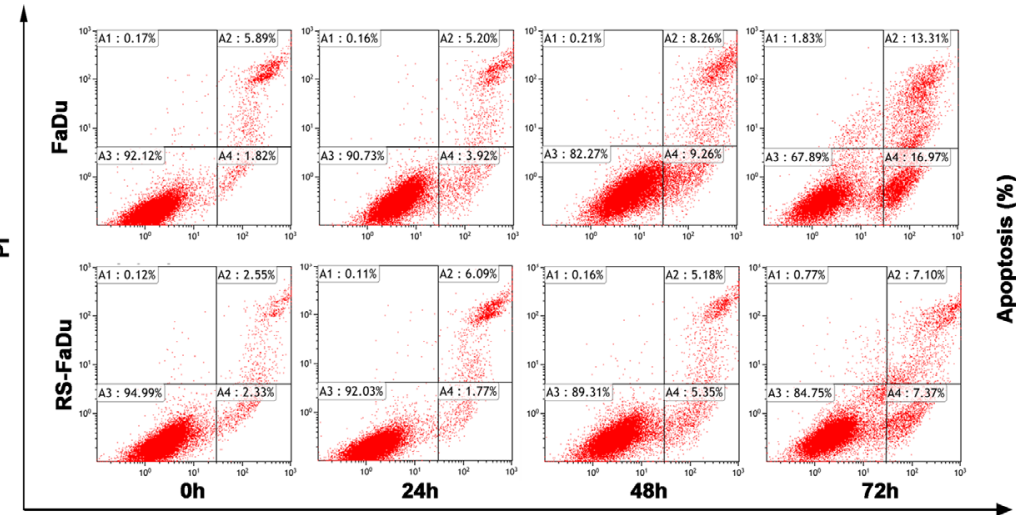

Annexin V

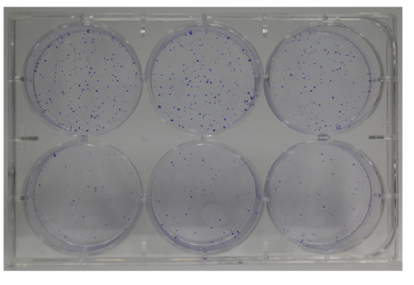

6Gy
B

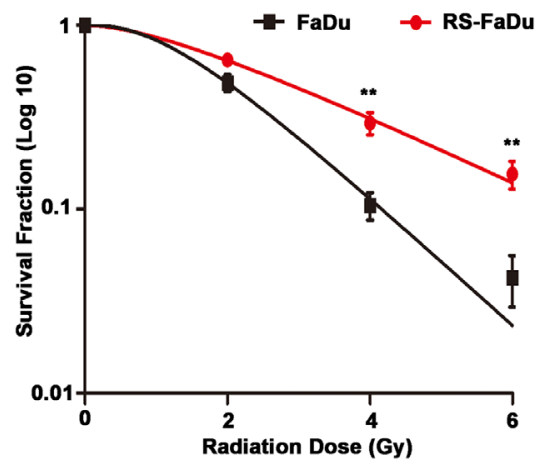

D

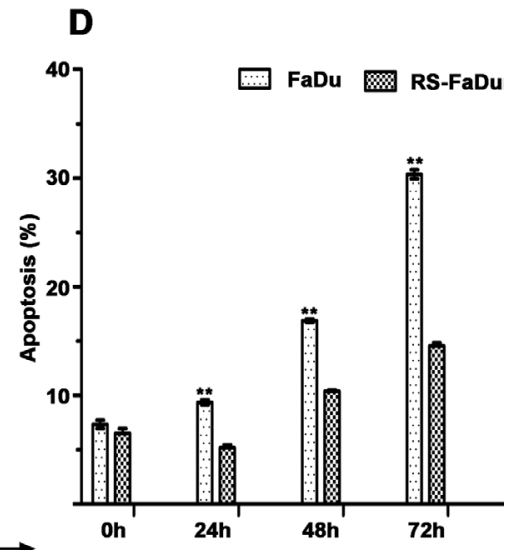

Figure 1: Radioresistance measurement by clonogenic survival assay and apoptosis assay. (A) RS-FaDu and parental FaDu cells irradiated with different radiation doses $(0,2,4$, and $6 \mathrm{~Gy})$ and the crystal violet-stained colonies were photographed at 12 days after irradiation. (B) Colonies containing more than 50 cells for survival colonies and scoring. (C) RS-FaDu and FaDu cells irradiated with 4 Gy. The apoptosis detection by FCM Annexin V/PI staining. The proportions of Annexin V+/PI- and Annexin V+/PI+ cells for earlyand late-stage apoptosis. (D) At $0 \mathrm{~h}$ after irradiation with $4 \mathrm{~Gy}$, there was no difference between RS-FaDu and FaDu in their fractions of apoptosis cells. At $24 \mathrm{~h}, 48 \mathrm{~h}$, or $72 \mathrm{~h}$ after irradiation, the fraction of apoptosis cells in RS-FaDu cells was lower than that in FaDu cells. All experiments were performed in triplicate wells; points, mean; bars, SD. ${ }^{* *} P<0.01$.
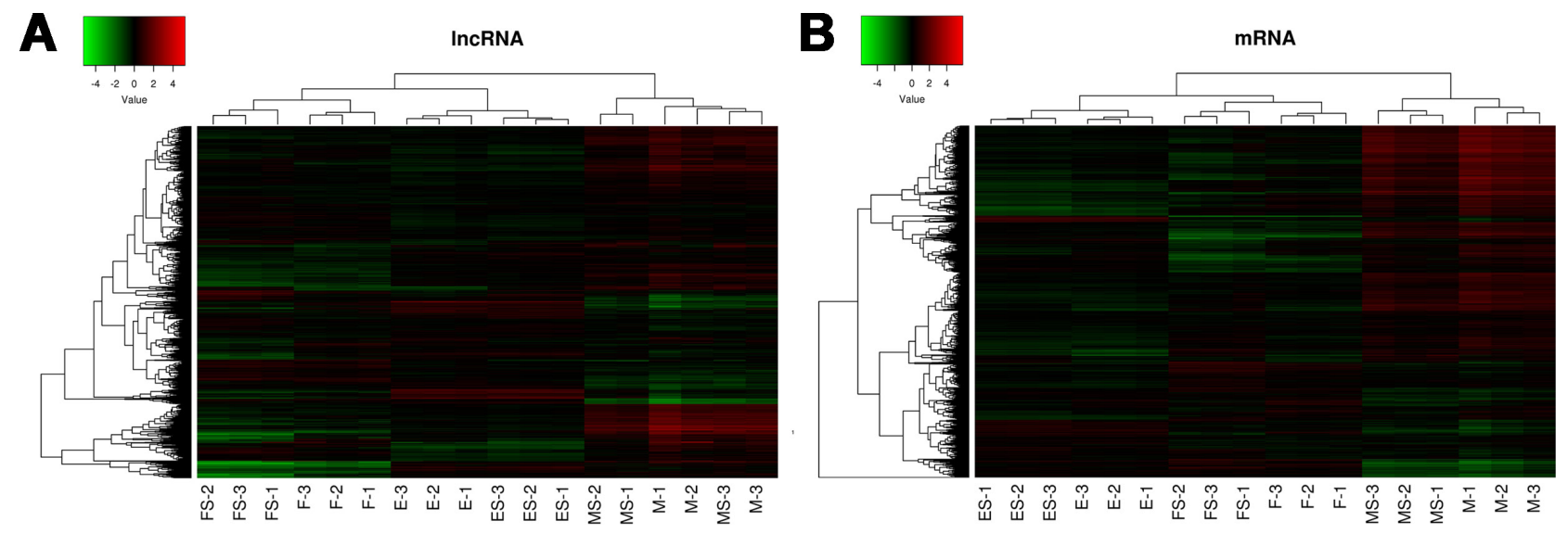

Figure 2: LncRNA (A) and mRNA (B) expression profiling by human lncRNA microarray. The sample tree on the top of the figure shows sample group information, which reflects relationships among samples. In the dendrogram, red indicates high relative expression, and blue indicates low relative expression. M, E, and F refer to $0 \mathrm{hr}, 2 \mathrm{hrs}$, and $48 \mathrm{hrs}$ after exposure to 4 Gy irradiation, respectively. The Arabic numerals presented the experiment repeats. 


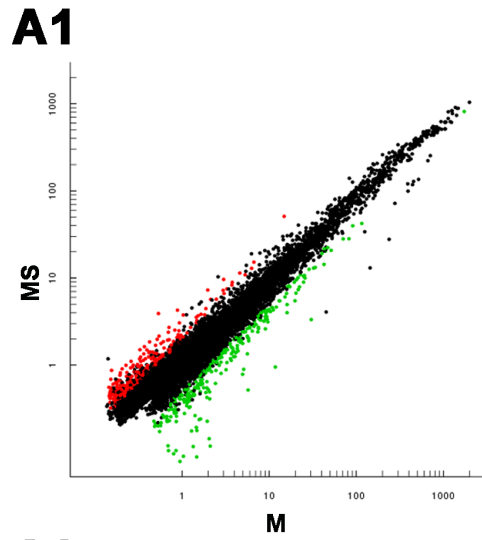

A2

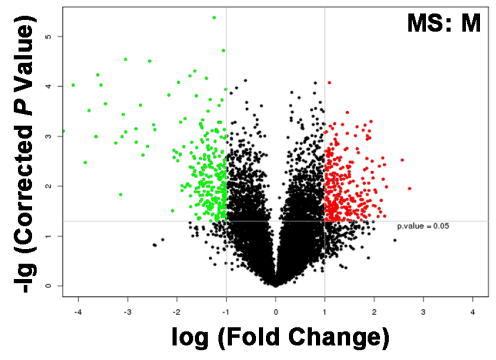

D1

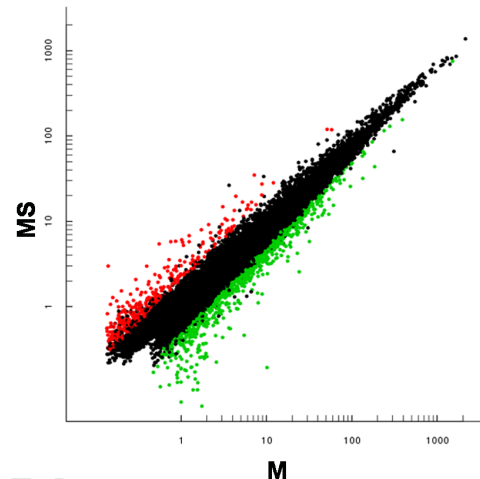

D2

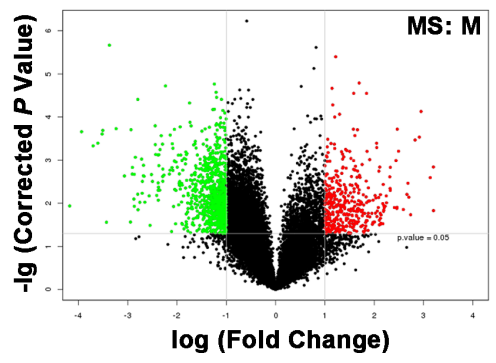

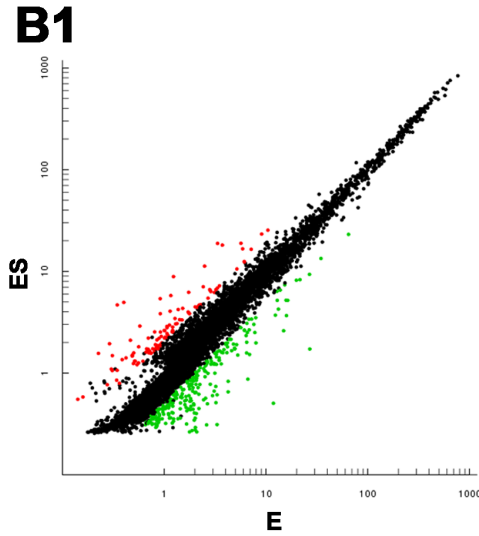

B2

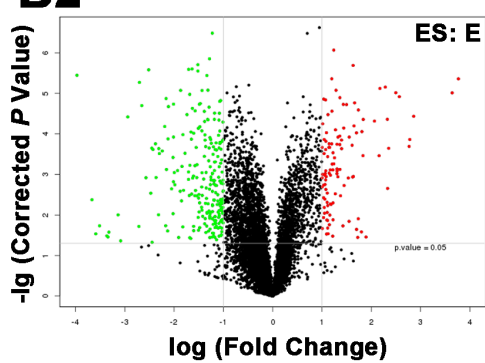

E1

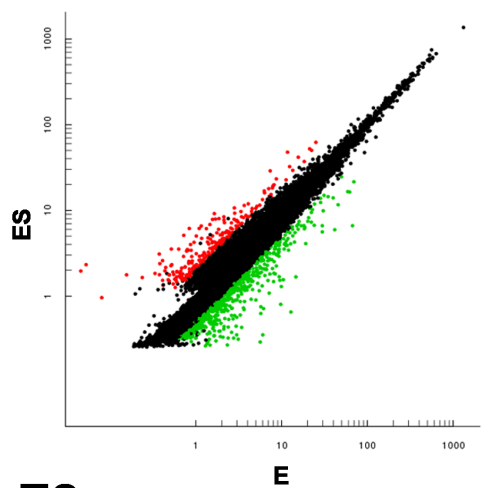

E2

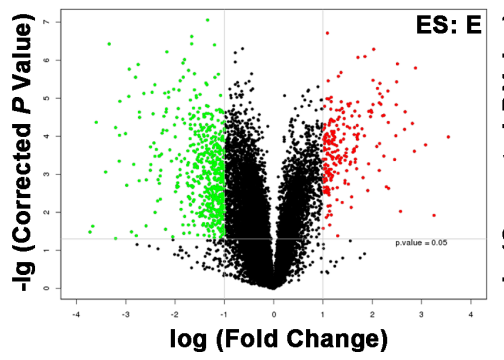

C1

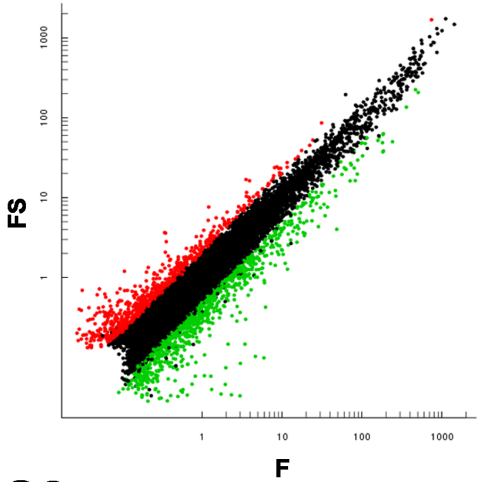

C2

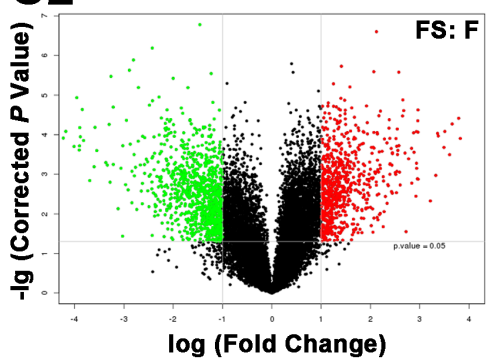

F1

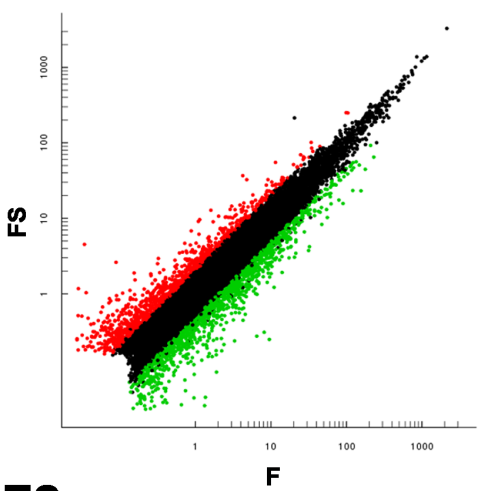

F2

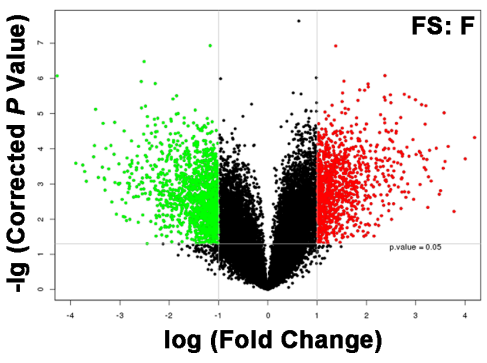

Figure 3: Differences in IncRNA and mRNA expression profiles between RS-FaDu cells and parental FaDu cells. (A1-F1) Scatter plots. Scatter plot showed the differentially expressed lncRNAs (A1-C1) and mRNAs (D1-F1). The values on the X and $\mathrm{Y}$ axes from the averaged normalized signal values of groups of samples ( $\log 2$ scaled). X-axis for control group ( $\mathrm{FaDu})$, and $\mathrm{Y}$-axis for case group (RS-FaDu). All the lncRNAs/mRNAs without differential expression around the line $\mathrm{Y}=\mathrm{X}$ in black. Points above $\mathrm{Y}=\mathrm{X}$ and apart from $\mathrm{Y}=\mathrm{X}$ are upregulated $\operatorname{lncRNAs} / \mathrm{mRNAs}$ in red, and points below $\mathrm{Y}=\mathrm{X}$ and apart from $\mathrm{Y}=\mathrm{X}$ are downregulated $\operatorname{lncRNAs} / \mathrm{mRNAs}$ in green, respectively. The threshold for differentially expressed genes was set at $F C \geq 2.0$. (A2-F2) Volcano plots for the differences in IncRNA (A2-C2) and mRNA (D2-F2) expression between the case group (RS-FaDu) and control group (FaDu). The X-axis in the volcano plot represents FC (after $\log 2$ transformation) and the Y-axis in the plot represents $P$-value (after log transformation). The $\operatorname{lncRNAs} / \mathrm{mRNAs}$ on the top left show downregulated lncRNAs/mRNAs ( $\mathrm{FC} \geq 2.0, P<0.05$ ). The $\operatorname{lncRNAs} / \mathrm{mRNAs}$ on the top right show upregulated lncRNAs/mRNAs ( $F C \geq 2.0, P<0.05$ ). Upregulated $\operatorname{lncRNAs} / \mathrm{mRNAs}$ and downregulated lncRNAs/mRNAs are shown in red and green, respectively. MS vs. M, 0 h; ES vs. E, 2 h; FS vs. F, 48 h. 
downregulated lncRNAs (Table 1) and mRNAs (Table 2) in $\mathrm{RS}-\mathrm{FaDu}$ cells at 0,2 , and $48 \mathrm{~h}$ after 4 Gy radiation, respectively.

Venn diagrams of the numbers and percentages of differentially expressed genes are shown in Figure 4. The results showed that there were $20(2.0 \%)$ common differentially upregulated lncRNAs (Figure 4A) and 65 (5.4\%) common differentially downregulated lncRNAs (Figure 4B) for the three groups (Supplementary Table 1). In addition, the numbers of common upregulated (Figure 4C) and downregulated (Figure 4D) differentially expressed mRNAs were $59(4.3 \%)$ and 153 (7.0\%), respectively (Supplementary Table 2).

\section{Validation of differential IncRNA expression by qRT-PCR}

The qRT-PCR was used to confirm the reliability and validity of microarray data. We selected four lncRNAs (ENST00000470135, TCONS_00010875, TCONS_00018436, and hox-HOXD10-35) for validation since these lncRNAs had consistent up- or downregulations at the three time points and their FC values were prominent at some time points. Additionally, four mRNAs (CKMT1A, GPNMB, FBLN5, and GDA) were validated as well due likely to their potential roles in irradiation response or radioresistance. The relative expression levels of the target RNAs were given as ratios of $\beta$-actin transcript levels in the same RNA samples. As shown in Figure 5, the expression levels of these eight genes were consistent with the microarray results, indicating the reliability of the microarray data and correlation of these genes with radioresistance.

\section{Potential roles of TCONS_00018436 in regulation of radioresistance of $\mathrm{HSCC}$}

We then assessed the expression of the four validated lncRNAs in primary tumor tissues of HSCC patients versus their recurrent ones after postoperative radiotherapy, using qRT-PCR. The expression of the three lncRNAs showed no remarkable difference between two groups of samples (Figure 6A-6C), while the significant upregulation of TCONS_00018436 in relapsed tumor samples was found (Figure 6D, $* P<0.05$ ). Further, after we stably knocked down TCONS 00018436 in FaDu-RS cells using lentiviral transfection, both transfected FaDuRS cells (FaDu-RS-sh) and FaDu-RS cells were treated with 4 Gy, 6 Gy and 8 Gy irradiation, respectively. The apoptotic cells at $48 \mathrm{~h}$ after irradiation were determined by Annexin V-FITC/PI and flow cytometry. As shown in Figure 6E, depletion of TCONS_00018436 significantly sensitized RS-FaDu cells to the indicated doses of radiation $\left({ }^{*} P<0.05\right)$, indicating that upregulated TCONS_00018436 might control radioresistance of HSCC cells during exposure to radiation. However, the underlying mechanism remains to be further investigated.

\section{Bioinformatics analyses}

In order to identify potential protein regulators involved in radioresistance of HSCC, we performed pathway enrichment analysis by functionally annotating and differentially expressed mRNAs in FaDu vs. FaDuRS cells at 0,2 , and $48 \mathrm{~h}$ after irradiation. The detailed information on significantly enriched pathway terms and dysregulated mRNAs at the three time points involved in them was presented in Supplementary Tables 3-5, respectively. The most significant 30 terms sorted by corrected $P$-value were separately listed in three histograms, according to the time points after irradiation shown in Supplementary Figure 1. This analysis approach may help identify altered expression of mRNAs involved in pathways associated with radioresistance, whose dysregulation might have an impact on sensitivity of HSCC to radiation.

An increasing number of lncRNAs have been shown to regulate expression of target genes in cis or in trans. Thus, we found significant correlations between dysregulated lncRNAs and mRNAs at 0, 2, and $48 \mathrm{~h}$ after irradiation, respectively. After lncRNA and mRNA correlation, cis-prediction and trans-prediction were both applied to the data. The final lncRNA prediction results are the combination of results from these two prediction parts at 0,2 , and $48 \mathrm{~h}$ as shown in Tables 3-5, respectively. These pathway enrichment analysis may provide some mRNA candidates that are potentially associated with radioresistance. Furthermore, we might further identify the lncRNAs in regulating the expressions of nearby or distant genes which encode these mRNAs based on the prediction results.

\section{DISCUSSION}

To investigate the role of IncRNAs in the radioresistance of $\mathrm{HSCC}$, we first observed the expression profiles of lncRNA in our established radioresistant HSCC cell model, i.e. RS-FaDu. The expression levels of lncRNA in the RS-FaDu and the parental FaDu cells were determined by microarray analysis immediately, at $2 \mathrm{~h}$ or $48 \mathrm{~h}$ after exposure to $4 \mathrm{~Gy}$ irradiation. This approach enabled us to observe the time-course differential expression patterns of IncRNA and mRNA in RS-FaDu $v s$. parental FaDu cells at the early and late stages of their irradiation response. Extracellular stimulation can bring about a rapid change on transcription of related genes. However, it is hard to determine the exact time points of each stage. Borràs-Fresneda, et al. found that a greatly differential transcriptional response in the radioresistant cell line was induced at $4 \mathrm{~h}$ after irradiation compared with the radiosensitive one [27]. To make transcriptomic analyses of the radiation response in head and neck squamous cell carcinoma subclones with different radiosensitivity, Michna, et al. even detected gene expression at $0.25,2,7,12,24,48,72$ and $96 \mathrm{~h}$ 
Table 1: Ten most upregulated and downregulated IncRNAs in RS-FaDu cells at 0, 2, and $48 \mathrm{~h}$ after 4 Gy radiation

\begin{tabular}{|c|c|c|c|c|c|c|c|c|}
\hline Probe name & FC (abs) & Regulation & IncRNA ID & $\mathrm{Chr}$ & Strand & Gene & Class & Time \\
\hline p7538 & 6.58 & up & ENST00000587434.1 & 17 & + & ENSG00000267601.1 & Antisense & $0 \mathrm{~h}$ \\
\hline p28462 & 5.94 & up & ASO3704 & & & ASO3704 & Intergenic & $0 \mathrm{~h}$ \\
\hline p8064 & 4.75 & up & ENST00000563172.1 & 18 & + & ENSG00000261780.2 & Intergenic & $0 \mathrm{~h}$ \\
\hline p19771 & 4.63 & up & TCONS_00023442 & 15 & + & XLOC_011287 & Intergenic & $0 \mathrm{~h}$ \\
\hline p37817_v4 & 4.62 & up & ENST00000607175.1 & 6 & - & ENSG00000272468.1 & & $0 \mathrm{~h}$ \\
\hline p35075_v4 & 4.54 & up & ENST00000594101.1 & 3 & + & ENSG00000242086.3 & Intergenic & $0 \mathrm{~h}$ \\
\hline p35072_v4 & 4.51 & up & ENST00000597871.1 & 3 & + & ENSG00000242086.3 & Intergenic & $0 \mathrm{~h}$ \\
\hline p11909 & 4.41 & up & ENST00000519700.1 & 3 & + & ENSG00000242770.2 & Intergenic & $0 \mathrm{~h}$ \\
\hline p6707 & 4.38 & up & ENST00000578710.1 & 17 & - & ENSG00000264673.1 & Intergenic & $0 \mathrm{~h}$ \\
\hline p35069_v4 & 4.30 & up & ENST00000438608.1 & 3 & + & ENSG00000242086.3 & Intergenic & $0 \mathrm{~h}$ \\
\hline p33918 & 19.81 & down & hox-HOXD10-35 & 2 & + & hox-HOXD10-35 & Intronic & $0 \mathrm{~h}$ \\
\hline p33919 & 17.23 & down & hox-HOXD10-36 & 2 & + & hox-HOXD10-36 & Intronic & $0 \mathrm{~h}$ \\
\hline p28077 & 14.53 & down & nc-HOXD10-9 & 2 & + & nc-HOXD10-9 & Intronic & $0 \mathrm{~h}$ \\
\hline p5033 & 13.80 & down & ENST00000556653.1 & 14 & + & ENSG00000258914.1 & Intergenic & $0 \mathrm{~h}$ \\
\hline p28072 & 12.49 & down & nc-HOXD10-13 & 2 & + & nc-HOXD10-13 & Intronic & $0 \mathrm{~h}$ \\
\hline p10912 & 12.20 & down & ENST00000430181.1 & 21 & + & ENSG00000235890.1 & Intronic & $0 \mathrm{~h}$ \\
\hline p28071 & 11.64 & down & nc-HOXD10-12 & 2 & + & nc-HOXD10-12 & Intronic & $0 \mathrm{~h}$ \\
\hline p6908 & 10.93 & down & ENST00000433510.1 & 17 & - & ENSG00000233283.2 & Intergenic & $0 \mathrm{~h}$ \\
\hline p8814 & 9.47 & down & ENST00000601506.1 & 19 & + & ENSG00000269495.1 & Antisense & $0 \mathrm{~h}$ \\
\hline p33495 & 8.82 & down & ENST00000589927.1 & 19 & + & ENSG00000186526.7 & Antisense & $0 \mathrm{~h}$ \\
\hline p29588 & 13.61 & up & TCONS_00018436 & 10 & - & XLOC_008730 & Intergenic & $2 \mathrm{~h}$ \\
\hline p29587 & 12.47 & up & TCONS_00017927 & 10 & - & XLOC_008730 & Intergenic & $2 \mathrm{~h}$ \\
\hline p22664 & 7.26 & up & TCONS_00010875 & 5 & - & XLOC_004700 & Intergenic & $2 \mathrm{~h}$ \\
\hline p5789 & 6.87 & up & ENST00000567091.1 & 16 & - & ENSG00000260394.2 & Divergent & $2 \mathrm{~h}$ \\
\hline p3381 & 6.81 & up & ENST00000547963.1 & 12 & - & ENSG00000249550.2 & Intergenic & $2 \mathrm{~h}$ \\
\hline p3379 & 5.93 & up & ENST00000550905.1 & 12 & - & ENSG00000249550.2 & Intergenic & $2 \mathrm{~h}$ \\
\hline p5993 & 5.64 & up & ENST00000567668.1 & 16 & - & ENSG00000260609.1 & Intergenic & $2 \mathrm{~h}$ \\
\hline p19771 & 5.11 & up & TCONS_00023442 & 15 & + & XLOC_011287 & Intergenic & $2 \mathrm{~h}$ \\
\hline p737 & 5.03 & up & ENST00000453572.1 & 1 & - & ENSG00000232184.1 & Intronic & $2 \mathrm{~h}$ \\
\hline p22663 & 5.01 & up & TCONS_00010233 & 5 & - & XLOC_004700 & Intergenic & $2 \mathrm{~h}$ \\
\hline p8814 & 23.31 & down & ENST00000601506.1 & 19 & + & ENSG00000269495.1 & Antisense & $2 \mathrm{~h}$ \\
\hline p8817 & 15.69 & down & ENST00000596286.1 & 19 & + & ENSG00000268739.1 & Antisense & $2 \mathrm{~h}$ \\
\hline p28076 & 12.71 & down & nc-HOXD10-8 & 2 & + & nc-HOXD10-8 & Antisense & $2 \mathrm{~h}$ \\
\hline p33919 & 12.04 & down & hox-HOXD10-36 & 2 & + & hox-HOXD10-36 & Intronic & $2 \mathrm{~h}$ \\
\hline p33918 & 11.42 & down & hox-HOXD10-35 & 2 & + & hox-HOXD10-35 & Intronic & $2 \mathrm{~h}$ \\
\hline p28072 & 10.30 & down & nc-HOXD10-13 & 2 & + & nc-HOXD10-13 & Intronic & $2 \mathrm{~h}$ \\
\hline p33495 & 10.15 & down & ENST00000589927.1 & 19 & + & ENSG00000186526.7 & Antisense & $2 \mathrm{~h}$ \\
\hline p28071 & 10.01 & down & nc-HOXD10-12 & 2 & + & nc-HOXD10-12 & Intronic & $2 \mathrm{~h}$ \\
\hline p8609 & 8.80 & down & ENST00000595892.1 & 19 & + & ENSG00000269640.1 & Divergent & $2 \mathrm{~h}$ \\
\hline p28077 & 8.48 & down & nc-HOXD10-9 & 2 & + & nc-HOXD10-9 & Intronic & $2 \mathrm{~h}$ \\
\hline p40301_v4 & 21.79 & up & XR_427456.1 & 3 & + & & & $48 \mathrm{~h}$ \\
\hline p3438 & 14.16 & up & ENST00000545853.1 & 12 & - & ENSG00000256732.1 & Intergenic & $48 \mathrm{~h}$ \\
\hline p18725 & 13.84 & up & TCONS_00020973 & 12 & - & XLOC_010243 & Intergenic & $48 \mathrm{~h}$ \\
\hline p33351 & 12.63 & up & ENST00000420462.1 & 1 & - & ENSG00000242663.1 & Antisense & $48 \mathrm{~h}$ \\
\hline p11893 & 12.18 & up & ENST00000462011.1 & 3 & + & ENSG00000244464.1 & Intergenic & $48 \mathrm{~h}$ \\
\hline p37817_v4 & 11.39 & up & ENST00000607175.1 & 6 & - & ENSG00000272468.1 & & $48 \mathrm{~h}$ \\
\hline p26490 & 11.25 & up & uc004aej.3 & 9 & - & BC065763 & Intergenic & $48 \mathrm{~h}$ \\
\hline p26072 & 10.39 & up & uc002oet. 3 & 19 & + & BC024306 & Intergenic & $48 \mathrm{~h}$ \\
\hline p29587 & 10.28 & up & TCONS_00017927 & 10 & - & XLOC_008730 & Intergenic & $48 \mathrm{~h}$ \\
\hline p14418 & 10.03 & up & ENST00000584911.1 & 6 & - & ENSG00000223414.2 & Intergenic & $48 \mathrm{~h}$ \\
\hline p28077 & 88.86 & down & nc-HOXD10-9 & 2 & + & nc-HOXD10-9 & Intronic & $48 \mathrm{~h}$ \\
\hline p33919 & 69.03 & down & hox-HOXD10-36 & 2 & + & hox-HOXD10-36 & Intronic & $48 \mathrm{~h}$ \\
\hline p8814 & 52.88 & down & ENST00000601506.1 & 19 & + & ENSG00000269495.1 & Antisense & $48 \mathrm{~h}$ \\
\hline p28072 & 49.57 & down & nc-HOXD10-13 & 2 & + & nc-HOXD10-13 & Intronic & $48 \mathrm{~h}$ \\
\hline p28076 & 45.45 & down & nc-HOXD10-8 & 2 & + & nc-HOXD10-8 & Antisense & $48 \mathrm{~h}$ \\
\hline p28071 & 41.28 & down & nc-HOXD10-12 & 2 & + & nc-HOXD10-12 & Intronic & $48 \mathrm{~h}$ \\
\hline p33495 & 38.99 & down & ENST00000589927.1 & 19 & + & ENSG00000186526.7 & Antisense & $48 \mathrm{~h}$ \\
\hline p33920 & 33.10 & down & hox-HOXD11-34 & 2 & + & hox-HOXD11-34 & Intronic & $48 \mathrm{~h}$ \\
\hline p 25262 & 26.48 & down & XR_108533.1 & 3 & - & LOC100505902 & Intergenic & $48 \mathrm{~h}$ \\
\hline p33918 & 23.62 & down & hox-HOXD10-35 & 2 & + & hox-HOXD10-35 & Intronic & $48 \mathrm{~h}$ \\
\hline
\end{tabular}


Table 2: Ten most upregulated and downregulated mRNAs in RS-FaDu cells at 0,2 and $48 \mathrm{~h}$ after 4 Gy radiation

\begin{tabular}{|c|c|c|c|c|c|}
\hline Probe name & FC (abs) & Regulation & Genbank accession & Gene symbol & Time \\
\hline A_21_P0005630 & 23.64873 & up & NR_121672 & LINC00824 & $0 \mathrm{~h}$ \\
\hline A_33_P3258346 & 9.20869 & up & NM_017523 & XAF1 & $0 \mathrm{~h}$ \\
\hline A_33_P3384287 & 9.20060 & up & NM_002579 & PALM & $0 \mathrm{~h}$ \\
\hline A_33_P3238533 & 8.80111 & up & NM_001105528 & CCDC178 & $0 \mathrm{~h}$ \\
\hline A_23_P87013 & 7.73073 & up & NM_001001522 & TAGLN & $0 \mathrm{~h}$ \\
\hline A_33_P3381948 & 7.55051 & up & NM_001080436 & WTIP & $0 \mathrm{~h}$ \\
\hline A_23_P1029 & 7.11102 & up & NM_017459 & MFAP2 & $0 \mathrm{~h}$ \\
\hline A_33_P3640690 & 6.67747 & up & NM_001128128 & ZEB1 & $0 \mathrm{~h}$ \\
\hline A_24_P557479 & 6.51224 & up & NM_017523 & XAF1 & $0 \mathrm{~h}$ \\
\hline A_33_P3237552 & 6.43079 & up & NM_032843 & FIBCD1 & $0 \mathrm{~h}$ \\
\hline A_33_P3290780 & 52.36956 & down & NM_001185156 & IL24 & $0 \mathrm{~h}$ \\
\hline A_33_P3260654 & 26.23980 & down & EU030678 & & $0 \mathrm{~h}$ \\
\hline A_24_P684183 & 18.09698 & down & NM_025257 & SLC44A4 & $0 \mathrm{~h}$ \\
\hline A_23_P304897 & 15.30950 & down & NM_000623 & BDKRB2 & $0 \mathrm{~h}$ \\
\hline A_23_P128744 & 13.02192 & down & NM_000710 & BDKRB1 & $0 \mathrm{~h}$ \\
\hline A_23_P122937 & 12.17637 & down & NM_014800 & ELMO1 & $0 \mathrm{~h}$ \\
\hline A_21_P0009192 & 11.46747 & down & & & $0 \mathrm{~h}$ \\
\hline A_23_P65189 & 11.36208 & down & NM_000209 & PDX1 & $0 \mathrm{~h}$ \\
\hline A_23_P39315 & 10.78665 & down & NM_021187 & CYP4F11 & $0 \mathrm{~h}$ \\
\hline A_23_P404494 & 10.34955 & down & NM_002185 & IL7R & $0 \mathrm{~h}$ \\
\hline A_21_P0005630 & 73.61931 & up & NR_121672 & LINC00824 & $2 \mathrm{~h}$ \\
\hline A_33_P3238533 & 61.95869 & up & NM_001105528 & CCDC178 & $2 \mathrm{~h}$ \\
\hline A_23_P69030 & 11.65424 & up & NM_001850 & COL8A1 & $2 \mathrm{~h}$ \\
\hline A_33_P3245439 & 9.52713 & up & NM_001250 & CD40 & $2 \mathrm{~h}$ \\
\hline A_23_P209055 & 8.46069 & up & NM_001771 & CD22 & $2 \mathrm{~h}$ \\
\hline A_33_P3293675 & 7.34682 & up & NM_006598 & SLC12A7 & $2 \mathrm{~h}$ \\
\hline A_24_P917886 & 7.22717 & up & XM_006709947 & MUC5AC & $2 \mathrm{~h}$ \\
\hline A_33_P3382177 & 7.02720 & up & NM_003255 & TIMP2 & $2 \mathrm{~h}$ \\
\hline A_32_P530933 & 6.34961 & up & NM_015617 & PYGO1 & $2 \mathrm{~h}$ \\
\hline A_23_P159721 & 6.29696 & up & NM_004224 & GPR50 & $2 \mathrm{~h}$ \\
\hline A_33_P3393971 & 27.56643 & down & NM_000299 & PKP1 & $2 \mathrm{~h}$ \\
\hline A_23_P23296 & 24.91828 & down & NM_000299 & PKP1 & $2 \mathrm{~h}$ \\
\hline A_23_P143029 & 21.06592 & down & NM_021192 & HOXD11 & $2 \mathrm{~h}$ \\
\hline A_24_P245379 & 13.24701 & down & NM_002575 & SERPINB2 & $2 \mathrm{~h}$ \\
\hline A_33_P3220911 & 12.74436 & down & NM_004335 & BST2 & $2 \mathrm{~h}$ \\
\hline A_33_P3226810 & 12.12693 & down & NM_003810 & TNFSF 10 & $2 \mathrm{~h}$ \\
\hline A_23_P404494 & 10.63455 & down & NM_002185 & IL7R & $2 \mathrm{~h}$ \\
\hline A_24_P236935 & 10.10874 & down & NM_001012964 & KLK6 & $2 \mathrm{~h}$ \\
\hline A_21_P0011633 & 9.24221 & down & NM_000526 & KRT14 & $2 \mathrm{~h}$ \\
\hline A_23_P39315 & 9.24213 & down & NM_021187 & CYP4F11 & $2 \mathrm{~h}$ \\
\hline A_33_P3238533 & 132.03939 & up & NM_001105528 & $\mathrm{CCDC} 178$ & $48 \mathrm{~h}$ \\
\hline A_21_P0005630 & 41.69042 & up & NR_121672 & LINC00824 & $48 \mathrm{~h}$ \\
\hline A_23_P161190 & 30.52496 & up & NM_003380 & VIM & $48 \mathrm{~h}$ \\
\hline A_23_P70468 & 29.94054 & up & NM_012367 & OR2B6 & $48 \mathrm{~h}$ \\
\hline A_33_P3340014 & 18.30612 & up & NM_016157 & TRO & $48 \mathrm{~h}$ \\
\hline A_33_P3514487 & 16.05847 & up & NM_198481 & VSTM1 & $48 \mathrm{~h}$ \\
\hline A_24_P211849 & 13.71514 & up & NM_001166220 & TBX20 & $48 \mathrm{~h}$ \\
\hline A 23 P 421379 & 12.63051 & up & NM 000612 & IGF2 & $48 \mathrm{~h}$ \\
\hline A_23_P69030 & 12.08606 & up & NM_001850 & COL8A1 & $48 \mathrm{~h}$ \\
\hline A_23_P41804 & 11.93085 & up & NM_033120 & NKD2 & $48 \mathrm{~h}$ \\
\hline A_23_P39315 & 39.50620 & down & NM_021187 & CYP4F11 & $48 \mathrm{~h}$ \\
\hline A_23_P143029 & 38.05859 & down & NM_021192 & HOXD11 & $48 \mathrm{~h}$ \\
\hline A_23_P50710 & 31.23669 & down & NM_001082 & CYP4F2 & $48 \mathrm{~h}$ \\
\hline A_33_P3393971 & 26.30644 & down & NM_000299 & PKP1 & $48 \mathrm{~h}$ \\
\hline A_23_P23296 & 24.31587 & down & NM_000299 & PKP1 & $48 \mathrm{~h}$ \\
\hline A_24_P42693 & 23.02732 & down & NM_021187 & CYP4F11 & $48 \mathrm{~h}$ \\
\hline A_23_P108280 & 20.67472 & down & NM_023944 & CYP4F12 & $48 \mathrm{~h}$ \\
\hline A_24_P684183 & 19.34901 & down & NM_025257 & SLC44A4 & $48 \mathrm{~h}$ \\
\hline A_23_P138541 & 14.88679 & down & NM_003739 & AKR1C3 & $48 \mathrm{~h}$ \\
\hline A 23 P300781 & 13.48931 & down & NM 013316 & CNOT4 & $48 \mathrm{~h}$ \\
\hline
\end{tabular}



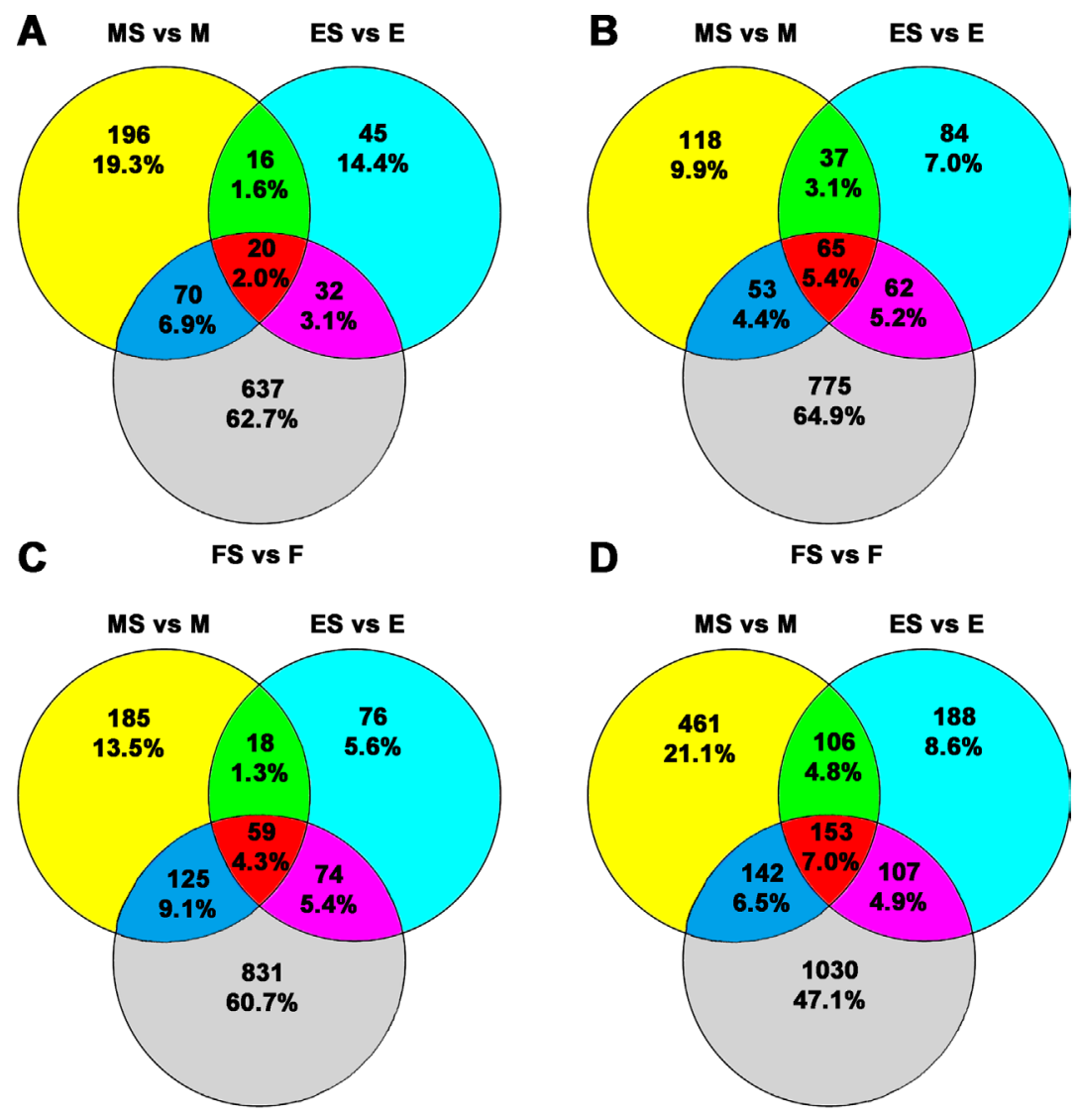

FS vs $F$

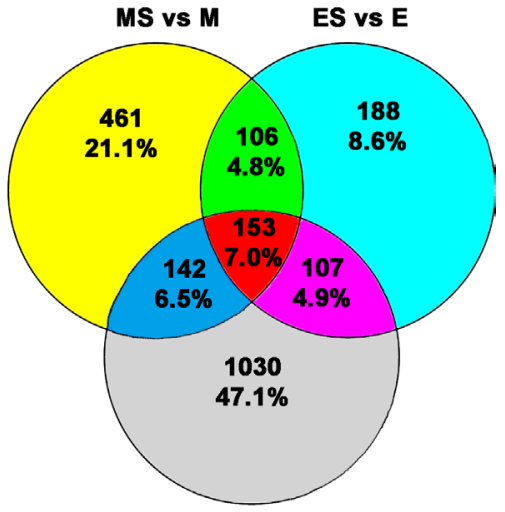

FS vs F

Figure 4: Venn diagram for the common and exclusively expressed IncRNAs and mRNAs from each group. FaDu and RS-FaDu cells at 0,2 , and 48 h after irradiation with 4 Gy. Different lncRNAs and mRNAs between FaDu and RS-FaDu as determined by microarray analysis for overlapping signature. (A) The overlapping results of upregulated differentially expressed lncRNAs. (B) The overlapping results of downregulated differentially expressed lncRNAs. (C) The overlapping results of upregulated differentially expressed mRNAs. (D) The overlapping results of downregulated differentially expressed mRNAs. MS vs. M, 0 h; ES vs. E, 2 h; FS vs. F, 48 h.
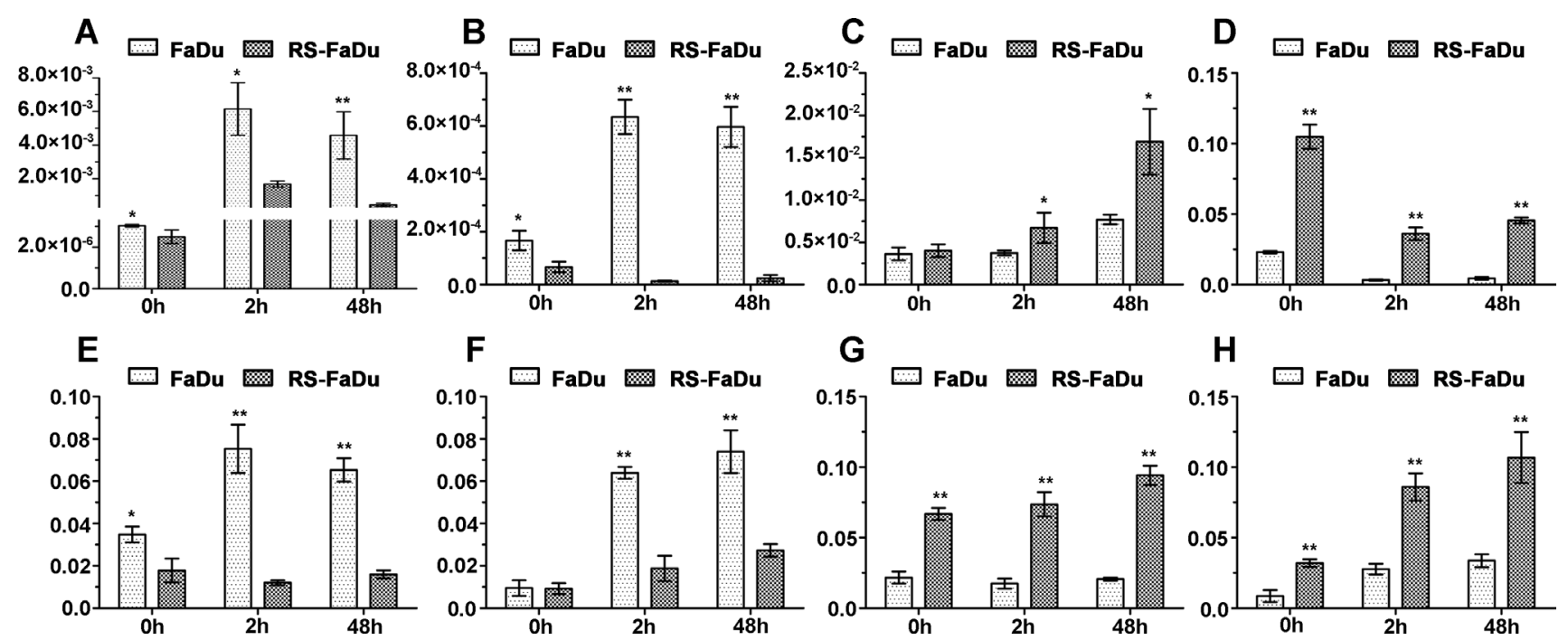

Figure 5: Validation of differential IncRNA expressions by qRT-PCR. (A) ENST00000470135; (B) hox-HOXD10-35; (C) TCONS_00010875; (D) TCONS_00018436; (E) CKMT1A; (F) GPNMB; (G) FBLN5; (H) GDA. After normalization to ACTB, data were presented as mean \pm SD. $n=3,{ }^{*} P<0.05, * * P<0.01$. 
after irradiation by microarray [28]. According to their experience, we selected these time points to roughly observe the early and late transcriptional response of $\mathrm{FaDu}$ and FaDu-RS cells to irradiation in the current study. Additionally, Li et al also carried out research on the relationship between lncRNA and radioresistance in nasopharyngeal carcinoma through genome-wide analyses [29]. However, the authors did not look at response time, which might effectively narrow our search for target lncRNAs and mRNAs.

Furthermore, we identified lncRNAs and mRNAs that were up- or downregulated at above three time points, and they were considered more likely to be involved in HSCC radioresistance. Subsequent validation experiments not only confirmed the reliability of the microarray data but also provided four lncRNA or mRNA candidates for our future mechanism study. Among these, TCONS_00018436 was considered more promising, due to that its potential role was preliminarily verified by its upregulated expression in relapsed tumor samples posterior to radiotherapy and loss-of-function assays.

Considering that altered response processes might occur at different stages after irradiation, we functionally annotated dysregulated mRNAs and performed bioinformatics analyses according to the respective response times. Among the most significantly enriched pathway terms, several attracted our attention because of their close relationship with radioresistance, such as the p53 signaling pathway at $0 \mathrm{~h}$ and at $2 \mathrm{~h}[30,31]$, and the Wnt signaling pathway at $48 \mathrm{~h}$ [32]. These results offer us preferential pathways in which to study the mechanisms underlying HSCC radioresistance. From these results, some candidate mRNAs could be identified based on their altered expression profiles of mRNAs as well.

LncRNAs are well known to affect the expression of target genes in cis or in trans through binding promoter regions of specific sequences, recruiting relevant transcription factors, and sequestering the interaction of miRNAs with target mRNAs, etc [33-36]. Thus, we identified dysregulated lncRNAs and their predicted mRNA targets on the basis of complementary base sequences and expression changes from our microarray data. According to dysregulation and association of mRNAs with radioresistance-related pathways in the pathway enrichment analysis, their matched lncRNAs could be found in our prediction results. We thought that it was likely a feasible way to search for lncRNA candidates for the further study.

Emerging evidence indicates that lncRNAs might function as competing endogenous RNAs by sponging miRNAs in a variety of cancers [37-40]. Notably, the lncRNAs NEAT1 [25] and MALAT1 [13, 24] have been shown to modulate radioresistance via sequestration of related miRNAs in nasopharyngeal carcinoma as well as high-risk human papillomavirus-positive cervical cancer. The regulatory roles of miRNAs are well established in the radioresistance of cancers [41-45], suggesting that we should explore the molecular mechanisms of lncRNAs in HSCC radioresistance from the perspective of lncRNAmiRNA-mRNA axes. Using sequence pairing, a number of dysregulated lncRNAs from our microarray data
A
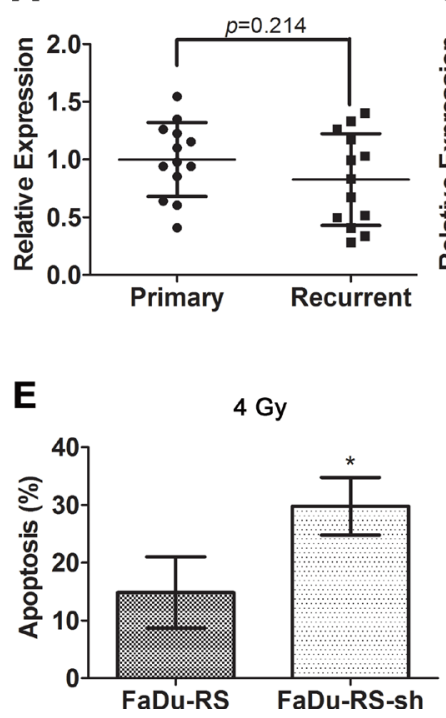

B

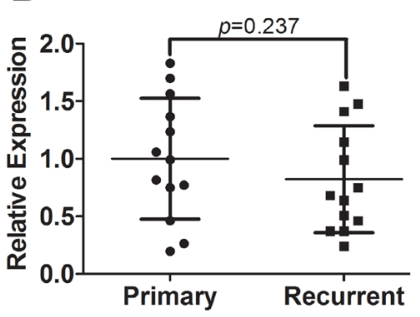

C

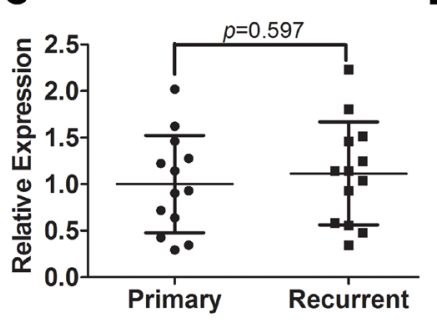

D

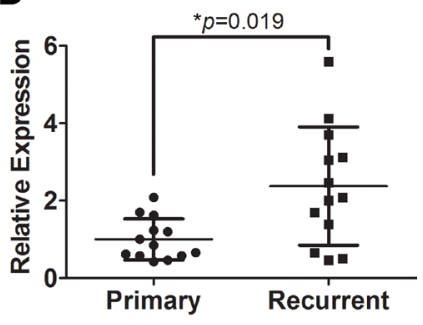

$6 \mathrm{~Gy}$

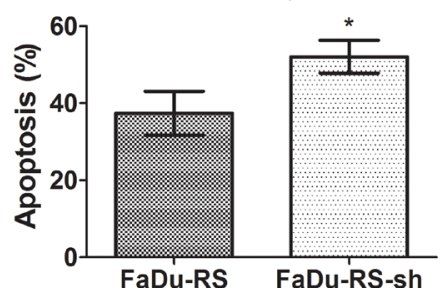

8 Gy

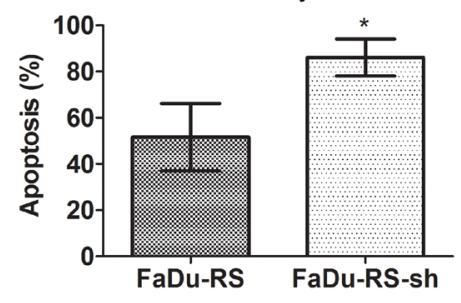

Figure 6: Potential roles of TCONS_00018436 in radioresistance of HSCC cells. The relative expression of ENST00000470135 (A), hox-HOXD10-35 (B), TCONS_00010875 (C) and TCONS_00018436 (D) in primary vs. recurrent HSCC tissue samples were measured by qRT-PCR. Their expression in each sample was normalized to the mean expression of their respective primary samples. Data were presented as mean \pm SD. $n=13, * P<0.05$. (E) FaDu-RS cells and those stably transfected with shRNA of TCONS_00018436 were both treated with $4 \mathrm{~Gy}, 6 \mathrm{~Gy}$, and $8 \mathrm{~Gy}$ irradiation, respectively. The fractions of apoptotic cells were determined by using Annexin V/PI dual staining after $48 \mathrm{~h}$. Data were presented as mean \pm SD. $n=3, * P<0.05$. 
Table 3: IncRNA target prediction of RS-FaDu vs. FaDu at $0 \mathrm{~h}$

\begin{tabular}{|c|c|c|c|c|c|c|}
\hline IncRNA & mRNA & Correlation & $P$-value & Direction (IncRNA-mRNA) & cisregulation & transregulation \\
\hline p3758 & A_33_P3421178 & 0.999026552 & 0.000001421 & down-down & Sense & \\
\hline p13056 & A_21_P0012973 & 0.993822774 & 0.000057119 & up-up & Sense & \\
\hline p25219 & A_33_P3368495 & 0.998540055 & 0.000003196 & down-down & & miRNA sequestration \\
\hline p7538 & A_33_P3382177 & 0.999529708 & 0.000000332 & up-up & Antisense & \\
\hline p35072_v4 & A_19_P00315941 & 0.997663892 & 0.000008180 & up-up & Sense & \\
\hline p12195 & A_19_P00316857 & 0.994153893 & 0.000051166 & up-up & Sense & \\
\hline p26166 & A_24_P557479 & -0.997108515 & 0.000012529 & down-up & & miRNA sequestration \\
\hline p29619 & A_21_P0007233 & 0.992506995 & 0.000084007 & down-down & Sense & \\
\hline p38695_v4 & A_23_P148919 & -0.99696864 & 0.000013770 & up-down & & miRNA sequestration \\
\hline p192 & A_23_P148919 & 0.994529457 & 0.000044808 & down-down & Antisense & \\
\hline p1665 & A_33_P3278211 & 0.994573678 & 0.000044088 & down-down & Sense & \\
\hline p30074 & A_33_P3289416 & 0.990995817 & 0.000121248 & down-down & & miRNA sequestration \\
\hline p10587 & A_24_P115199 & -0.99188824 & 0.000098434 & up-down & & miRNA sequestration \\
\hline p8323 & A_24_P115199 & -0.997557313 & 0.000008943 & up-down & & miRNA sequestration \\
\hline p687 & A_21_P0001239 & 0.991689858 & 0.000103301 & down-down & Sense & \\
\hline p686 & A_21_P0001239 & 0.999058836 & 0.000001328 & down-down & Sense & \\
\hline p34021_v4 & A_21_P0001239 & 0.998331716 & 0.000004172 & down-down & Sense & \\
\hline p7388 & A_33_P3341836 & 0.997644418 & 0.000008317 & up-up & & miRNA sequestration \\
\hline p687 & A_32_P212373 & 0.991025177 & 0.000120460 & down-down & Sense & \\
\hline p686 & A_32_P212373 & 0.998954092 & 0.000001640 & down-down & Sense & \\
\hline p34021_v4 & A_32_P212373 & 0.998577934 & 0.000003032 & down-down & Sense & \\
\hline p686 & A_21_P0001238 & 0.994752897 & 0.000041226 & down-down & Sense & \\
\hline p34021_v4 & A_21_P0001238 & 0.992305807 & 0.000088573 & down-down & Sense & \\
\hline p38695_v4 & A_33_P3414487 & -0.99790579 & 0.000006574 & up-down & & miRNA sequestration \\
\hline p10433 & A_23_P210425 & 0.993170163 & 0.000069811 & up-up & Antisense & \\
\hline p4963 & A_23_P304897 & 0.994892042 & 0.000039070 & down-down & Intronic & \\
\hline p6222 & A_24_P80135 & 0.997657113 & 0.000008227 & down-down & & miRNA sequestration \\
\hline p15211 & A_23_P320878 & 0.993544033 & 0.000062385 & down-down & & miRNA sequestration \\
\hline
\end{tabular}

and matched potential miRNA targets were identified (data not shown) in preparation for verifying our hypothesis.

In addition to the exploration of mechanisms underlying $\mathrm{HSCC}$ radioresistance, the identification of biomarkers for predicting radioresistant HSCC is of great clinical significance. Recently, a growing number of circulating or tissue-derived lncRNAs have been shown to be correlated with clinicopathological characteristics in patients with cancer [46-48], making them promising candidate biomarkers of malignancy. Given the close relationship between lncRNAs and radioresistant HSCC cells discussed above, lncRNAs have the potential to become novel biomarkers for the evaluation of HSCC radioresistance. We further plan to measure the expression levels of candidate lncRNAs in tumor tissues as well as blood samples from HSCC patients and explore the correlation between expression levels and different responses of HSCC patients to routine radiation therapy.

In this study, we for the first time have comprehensively demonstrated the time-course expression profiles of human IncRNAs/mRNAs in radioresistant $\mathrm{RS}-\mathrm{FaDu}$ cells derived from $\mathrm{FaDu}$ cells. Through validation experiments and subsequent preliminary investigation, TCONS_00018436 emerged as a promising candidate for studying the molecular mechanism underlying radioresistance of HSCC. Moreover, a large number of lncRNAs or mRNAs still awaits for being discovered through the bioinformatics analyses. In conclusion, our data laid the foundation for further investigating the roles of these IncRNAs and mRNAs in the occurrence and development of HSCC radioresistance. In addition, novel therapeutic targets and diagnostic biomarkers are likely to be identified in the future on the basis of our data.

\section{MATERIALS AND METHODS}

\section{Establishment of a radioresistant cell line}

The HSCC FaDu cell line was purchased from the Type Culture Collection of the Chinese Academy of Sciences (Beijing, China). The cells were cultured in MEM (Gibico, Grand Island, NY, USA) with 10\% fetal bovine serum (FBS), $2 \mathrm{mM}$ glutamine, 100 units $/ \mathrm{ml}$ penicillin, and $100 \mu \mathrm{g} / \mathrm{ml}$ streptomycin and incubated at $37^{\circ} \mathrm{C}$ with humidified $5 \% \mathrm{CO}_{2}$. 
Table 4: LncRNA target prediction of RS-FaDu vs. FaDu at 2 h

\begin{tabular}{|c|c|c|c|c|c|c|}
\hline IncRNA & mRNA & Correlation & $P$-value & Direction (IncRNA-mRNA) & cisregulation & transregulation \\
\hline p20305 & A_33_P3266898 & -0.996352571 & 0.000019931 & up-down & & miRNA sequestration \\
\hline p30194 & A_33_P3266898 & -0.996726605 & 0.000016055 & up-down & & miRNA sequestration \\
\hline p33576 & A_32_P131031 & 0.992702048 & 0.000079696 & down-down & Intergenic (10 k) & \\
\hline p36121_v4 & A_21_P0004245 & 0.990861638 & 0.000124883 & down-down & Sense & \\
\hline p8814 & A_24_P236935 & 0.995770285 & 0.000026798 & down-down & Antisense & \\
\hline p687 & A_24_P915692 & 0.990515655 & 0.000134503 & down-down & & miRNA sequestration \\
\hline p24838 & A_33_P3278211 & 0.998051663 & 0.000005690 & down-down & Sense & \\
\hline p1665 & A_33_P3278211 & 0.998814061 & 0.000002109 & down-down & Sense & \\
\hline p37870_v4 & A_33_P3289416 & -0.990915820 & 0.000123409 & up-down & & miRNA sequestration \\
\hline p25347 & A_24_P940149 & -0.996508538 & 0.000018264 & up-down & & miRNA sequestration \\
\hline p25347 & A_32_P152437 & 0.996042331 & 0.000023464 & up-up & & miRNA sequestration \\
\hline p30340 & A_24_P602871 & 0.995141025 & 0.000035357 & down-down & & miRNA sequestration \\
\hline p25605 & A_23_P329112 & 0.996929698 & 0.000014126 & up-down & & miRNA sequestration \\
\hline p25347 & A_23_P329112 & 0.994658656 & 0.000042719 & up-down & & miRNA sequestration \\
\hline p11034 & A_24_P66027 & 0.991916997 & 0.000097738 & down-down & Antisense & \\
\hline p28133 & A_24_P115199 & 0.997640377 & 0.000008345 & down-down & & miRNA sequestration \\
\hline p30155 & A_24_P115199 & 0.990576762 & 0.000132778 & down-down & & miRNA sequestration \\
\hline p33798 & A_23_P60339 & -0.994723656 & 0.000041686 & up-down & & miRNA sequestration \\
\hline p687 & A_32_P212373 & 0.992913328 & 0.000075153 & down-down & Sense & \\
\hline p686 & A_32_P212373 & 0.994692846 & 0.000042174 & down-down & Sense & \\
\hline p34021_v4 & A_32_P212373 & 0.991057378 & 0.000119598 & down-down & Sense & \\
\hline p20305 & A_23_P208389 & 0.996581866 & 0.000017505 & up-up & & miRNA sequestration \\
\hline p34907_v4 & A_33_P3333554 & 0.990605549 & 0.000131969 & down-down & & miRNA sequestration \\
\hline p10433 & A_23_P210425 & 0.998311004 & 0.000004277 & up-up & Antisense & \\
\hline p30335 & A_23_P157022 & 0.991277419 & 0.000113793 & down-down & & miRNA sequestration \\
\hline p15938 & A_21_P0005906 & 0.995291866 & 0.000033198 & down-down & Intergenic (10 k) & \\
\hline p34907_v4 & A_33_P3378430 & 0.991616881 & 0.000105120 & down-down & & miRNA sequestration \\
\hline p20305 & A_32_P31618 & -0.992612720 & 0.000081656 & up-down & & miRNA sequestration \\
\hline p15519 & A_33_P3233273 & 0.990760089 & 0.000127670 & down-down & Intergenic $(10 \mathrm{k})$ & \\
\hline
\end{tabular}

RS-FaDu cells were created by repeatedly exposing the parental FaDu cells to irradiation [15]. Briefly, FaDu cells were grown in $75-\mathrm{mm}^{2}$ cell culture plates. After the cells reached $70-80 \%$ confluence, they were irradiated with X-rays at room temperature. The X-ray generator (MBR-1505R; Hitachi Medical Co., Tokyo, Japan) was operated at $210 \mathrm{kV}$ and $10 \mathrm{~mA}$, with $0.5 \mathrm{~mm} \mathrm{Al}$ external filtration. The dose rate was $1.8 \mathrm{~Gy} / \mathrm{min}$. The cells were exposed to doses of $2,4,6,8$, and 10 Gy and were irradiated with each dose twice (total dose of $60 \mathrm{~Gy}$ ). An interval of 2 to 4 weeks between each dose allowed the surviving cells to regenerate. The process of irradiation and culture lasted for about 10 months. The HSCC cell clones that recovered after exposure to ionizing radiation were collected for further experiments.

\section{Patient specimens}

Primary and recurrent tumor samples were obtained from 13 patients who received radiotherapy followed by surgery in Qilu hospital from March 2013 to October 2015 , and then salvage surgery due to local recurrence. After surgery, samples were cleaned with phosphatebuffered saline (PBS) and immediately put into liquid nitrogen at once. At least $24 \mathrm{~h}$ later, samples were transferred to $-80^{\circ} \mathrm{C}$ for long-term storage. Characteristics of patients were summarized in Table 6. Prior to this study, written informed contents were signed, and this study was conducted under the approval of the institutional review board of the Ethics Boards of Qilu Hospital.

\section{Clonogenic assay}

Both parental $\mathrm{FaDu}$ and radioresistant $\mathrm{RS}-\mathrm{FaDu}$ cells were plated in six-well culture plates and irradiated with a single dose of $0,2,4$, or $6 \mathrm{~Gy}$, respectively. Plated cell numbers were as follows: 300 cells for $0 \mathrm{~Gy}, 600$ cells for $2 \mathrm{~Gy}, 900$ cells for $4 \mathrm{~Gy}$, and 1200 cells for $6 \mathrm{~Gy}$. Following irradiation, the cells were cultured in a $5 \%$ $\mathrm{CO}_{2}$ atmosphere at $37^{\circ} \mathrm{C}$, and the medium was changed every 3 days. After 12 days, colonies were fixed with ethanol for $15 \mathrm{~min}$ and stained with $0.1 \%$ crystal violet for 15 min. Colonies with $>50$ cells were scored with a ColCount colony counter (Oxford Optronix, Oxford, United Kingdom). All experiments were performed in triplicate. The survival fraction (SF) was estimated by the following formula: $\mathrm{SF}=$ [number of colonies formed/ number of cells seeded $\times$ plating efficiency of the control group], where plating efficiency was calculated as the ratio between colonies observed and number of cells plated. 
Table 5: LncRNA target prediction of RS-FaDu vs. FaDu at $48 \mathrm{~h}$

\begin{tabular}{|c|c|c|c|c|c|c|}
\hline IncRNA & mRNA & Correlation & $P$-value & Direction (IncRNA-mRNA) & cisregulation & transregulation \\
\hline p24892 & A_33_P3250133 & 0.993774158 & 0.000058021 & up-up & & miRNA sequestration \\
\hline p36462_v4 & A_24_P111242 & -0.997243502 & 0.000011387 & down-up & & miRNA sequestration \\
\hline p2627 & A_21_P0014268 & 0.996216336 & 0.000021447 & up-up & Sense & \\
\hline p25293 & A_23_P38813 & -0.997569809 & 0.000008852 & up-down & & miRNA sequestration \\
\hline p24618 & A_23_P38813 & 0.996136217 & 0.000022364 & down-down & & miRNA sequestration \\
\hline p37916_v4 & A_23_P38813 & -0.991608950 & 0.000105319 & up-down & & miRNA sequestration \\
\hline p44617_v4 & A_23_P38813 & -0.993806999 & 0.000057411 & up-down & & miRNA sequestration \\
\hline p25051 & A_23_P38813 & -0.995045168 & 0.000036765 & up-down & & miRNA sequestration \\
\hline p28498 & A_23_P38813 & -0.994453852 & 0.000046054 & up-down & & miRNA sequestration \\
\hline p122 & A_23_P38813 & -0.994491445 & 0.000045433 & up-down & & miRNA sequestration \\
\hline p3143 & A_23_P38813 & 0.997519222 & 0.000009224 & down-down & & miRNA sequestration \\
\hline p11437 & A_23_P38813 & -0.995453161 & 0.000030964 & up-down & & miRNA sequestration \\
\hline p37026_v4 & A_23_P38813 & -0.994529667 & 0.000044805 & up-down & & miRNA sequestration \\
\hline p23798 & A_23_P38813 & 0.992085159 & 0.000093719 & down-down & & miRNA sequestration \\
\hline p29606 & A_23_P38813 & -0.998223527 & 0.000004731 & up-down & & miRNA sequestration \\
\hline p28658 & A_23_P38813 & -0.993772836 & 0.000058046 & up-down & & miRNA sequestration \\
\hline p24771 & A_21_P0014060 & 0.995431495 & 0.000031259 & up-up & Sense & \\
\hline p34907_v4 & A_24_P319364 & -0.996770625 & 0.000015626 & down-up & & miRNA sequestration \\
\hline p29827 & A_33_P3336622 & 0.994560238 & 0.000044306 & up-up & & miRNA sequestration \\
\hline p29606 & A_33_P3336622 & 0.993785701 & 0.000057806 & up-up & & miRNA sequestration \\
\hline p26063 & A_33_P3390723 & 0.990423713 & 0.000137119 & up-up & & miRNA sequestration \\
\hline p24892 & A_23_P45799 & -0.997314248 & 0.000010810 & up-down & & miRNA sequestration \\
\hline p34907_v4 & A_24_P129232 & -0.992791851 & 0.000077749 & down-up & & miRNA sequestration \\
\hline p18271 & A_24_P684183 & -0.990582868 & 0.000132606 & up-down & & miRNA sequestration \\
\hline p44617_v4 & A_24_P684183 & -0.990879668 & 0.000124391 & up-down & & miRNA sequestration \\
\hline p18272 & A_24_P684183 & -0.993001983 & 0.000073287 & up-down & & miRNA sequestration \\
\hline p8313 & A_23_P165186 & 0.990188970 & 0.000143912 & up-up & Bidirectional & \\
\hline p7538 & A_33_P3382177 & 0.990676885 & 0.000129976 & up-up & Antisense & \\
\hline p41822_v4 & A_32_P126698 & 0.993882119 & 0.000056028 & up-up & & miRNA sequestration \\
\hline p25293 & A_23_P149099 & -0.991073247 & 0.000119175 & up-down & & miRNA sequestration \\
\hline p18271 & A_23_P149099 & -0.991096396 & 0.000118558 & up-down & & miRNA sequestration \\
\hline p823 & A_23_P149099 & -0.992591892 & 0.000082117 & up-down & & miRNA sequestration \\
\hline p23798 & A_23_P149099 & 0.993960353 & 0.000054606 & down-down & & miRNA sequestration \\
\hline p25999 & A_23_P149099 & -0.991209023 & 0.000115582 & up-down & & miRNA sequestration \\
\hline p25051 & A_24_P382630 & -0.998198512 & 0.000004865 & up-down & & miRNA sequestration \\
\hline p28498 & A_24_P382630 & -0.991372680 & 0.000111325 & up-down & & miRNA sequestration \\
\hline p16320 & A_23_P62679 & -0.995317469 & 0.000032838 & up-down & & miRNA sequestration \\
\hline p13683 & A_33_P3233841 & 0.993630385 & 0.000060729 & up-up & Antisense & \\
\hline p21313 & A_19_P00317856 & 0.993327189 & 0.000066641 & up-up & Sense & miRNA sequestration \\
\hline p36492_v4 & A_33_P3221748 & 0.995546108 & 0.000029712 & up-up & Sense & \\
\hline p19344 & A_24_P250535 & 0.991880869 & 0.000098613 & up-up & & miRNA sequestration \\
\hline p8693 & A_24_P250535 & 0.991653359 & 0.000104209 & up-up & & miRNA sequestration \\
\hline p14566 & A_19_P00319254 & 0.991633933 & 0.000104694 & down-down & Sense & \\
\hline p22664 & A_23_P41804 & 0.995620949 & 0.000028722 & up-up & Intergenic (10 k) & \\
\hline p22663 & A_23_P41804 & 0.997949994 & 0.000006299 & up-up & Intergenic (10 k) & \\
\hline p37916_v4 & A_33_P3215277 & 0.992325400 & 0.000088123 & up-up & & miRNA sequestration \\
\hline p28498 & A_33_P3215277 & 0.993349818 & 0.000066190 & up-up & & miRNA sequestration \\
\hline p20125 & A_33_P3256490 & 0.993114016 & 0.000070962 & up-up & & miRNA sequestration \\
\hline p2107 & A_33_P3256490 & 0.996526411 & 0.000018078 & up-up & & miRNA sequestration \\
\hline p33596 & A_23_P347468 & 0.990934613 & 0.000122899 & down-down & & miRNA sequestration \\
\hline $\mathrm{p} 4313$ & A_33_P3239084 & 0.991022154 & 0.000120541 & down-down & & miRNA sequestration \\
\hline p26099 & A_23_P381368 & 0.992698802 & 0.000079767 & down-down & Intergenic (10 k) & \\
\hline p28109 & A_23_P381368 & 0.998436798 & 0.000003663 & down-down & & miRNA sequestration \\
\hline p5755 & A_24_P56894 & 0.990801171 & 0.000126538 & up-up & & miRNA sequestration \\
\hline p16320 & A_24_P56894 & 0.991795931 & 0.000100684 & up-up & & miRNA sequestration \\
\hline p2195 & A_23_P134935 & 0.995584780 & 0.000029198 & up-up & & miRNA sequestration \\
\hline p18273 & A_23_P76749 & 0.991517681 & 0.000107619 & up-up & & miRNA sequestration \\
\hline p18273 & A_23_P336929 & 0.990945545 & 0.000122604 & up-up & & miRNA sequestration \\
\hline p3234 & A_24_P380022 & -0.990116941 & 0.000146030 & up-down & & miRNA sequestration \\
\hline p16320 & A_33_P3319593 & -0.991594493 & 0.000105682 & up-down & & miRNA sequestration \\
\hline p37870_v4 & A_33_P3319593 & -0.993768247 & 0.000058131 & up-down & & miRNA sequestration \\
\hline p36411_v4 & A_24_P941217 & -0.992930705 & 0.000074786 & down-up & & miRNA sequestration \\
\hline p25274 & A_24_P941217 & 0.993605918 & 0.000061196 & up-up & & miRNA sequestration \\
\hline p30340 & A_23_P209320 & -0.991624411 & 0.000104932 & down-up & & miRNA sequestration \\
\hline p34907_v4 & A_33_P3424297 & 0.991763098 & 0.000101490 & down-down & & miRNA sequestration \\
\hline p30340 & A_33_P3424297 & 0.990792516 & 0.000126776 & down-down & & miRNA sequestration \\
\hline p8323 & A_33_P3418394 & 0.996071022 & 0.000023125 & up-up & & miRNA sequestration \\
\hline p11437 & A_33_P3418394 & 0.992377207 & 0.000086939 & up-up & & miRNA sequestration \\
\hline p1517 & A_21_P0014248 & 0.993602194 & 0.000061267 & up-up & Sense & \\
\hline p14566 & A_19_P00322225 & 0.991838124 & 0.000099652 & down-down & Sense & \\
\hline p26790 & A_24_P940149 & 0.993717158 & 0.000059087 & down-down & Antisense & \\
\hline p17915 & A_23_P416305 & -0.993783331 & 0.000057850 & down-up & & miRNA sequestration \\
\hline
\end{tabular}




\begin{tabular}{|c|c|c|c|c|c|c|}
\hline p750 & A_21_P0010797 & 0.996064600 & 0.000023201 & up-up & Sense & \\
\hline p29253 & A_32_P173058 & 0.992308714 & 0.000088506 & up-up & & miRNA sequestration \\
\hline p36497_v4 & A_19_P00319372 & 0.993618413 & 0.000060957 & up-up & Intronic & \\
\hline p36069_v4 & A_19_P00319372 & 0.994980191 & 0.000037734 & up-up & Sense & \\
\hline p3143 & A_23_P383031 & 0.994487956 & 0.000045490 & down-down & & miRNA sequestration \\
\hline p18271 & A_24_P409042 & -0.993703568 & 0.000059343 & up-down & & miRNA sequestration \\
\hline p24618 & A_24_P409042 & 0.997488035 & 0.000009457 & down-down & & miRNA sequestration \\
\hline p18273 & A_24_P409042 & -0.991108770 & 0.000118230 & up-down & & miRNA sequestration \\
\hline $\mathrm{p} 25051$ & A_24_P409042 & -0.994189287 & 0.000050548 & up-down & & miRNA sequestration \\
\hline p18272 & A_24_P409042 & -0.994084150 & 0.000052392 & up-down & & miRNA sequestration \\
\hline p7539 & A_24_P409042 & -0.991072129 & 0.000119205 & up-down & & miRNA sequestration \\
\hline p18273 & A_23_P397856 & -0.993936073 & 0.000055045 & up-down & & miRNA sequestration \\
\hline p6823 & A_24_P254346 & -0.995095345 & 0.000036024 & up-down & & miRNA sequestration \\
\hline p41766_v4 & A_23_P97021 & -0.991498540 & 0.000108105 & up-down & & miRNA sequestration \\
\hline p29786 & A_23_P97021 & -0.995760159 & 0.000026926 & up-down & & miRNA sequestration \\
\hline p9299 & A_23_P97021 & -0.990583844 & 0.000132579 & up-down & & miRNA sequestration \\
\hline $\mathrm{p} 28283$ & A_23_P97021 & -0.991229915 & 0.000115034 & up-down & & miRNA sequestration \\
\hline p18271 & A_24_P373286 & 0.992099626 & 0.000093377 & up-up & & miRNA sequestration \\
\hline p16298 & A_32_P90080 & 0.997392142 & 0.000010193 & down-down & & miRNA sequestration \\
\hline p7391 & A_23_P371885 & 0.995164968 & 0.000035010 & up-up & & miRNA sequestration \\
\hline p16320 & A_21_P0013080 & -0.991724549 & 0.000102441 & up-down & & miRNA sequestration \\
\hline p37870_v4 & A_21_P0013080 & -0.990354772 & 0.000139097 & up-down & & miRNA sequestration \\
\hline p4547 & A_23_P329112 & -0.990672972 & 0.000130084 & down-up & & miRNA sequestration \\
\hline p11094 & A_23_P26704 & -0.990068457 & 0.000147464 & up-down & & miRNA sequestration \\
\hline p8313 & A_23_P26704 & -0.992380572 & 0.000086862 & up-down & & miRNA sequestration \\
\hline p29606 & A_23_P26704 & -0.996163250 & 0.000022053 & up-down & & miRNA sequestration \\
\hline p15693 & A_23_P304682 & -0.990624661 & 0.000131433 & up-down & & miRNA sequestration \\
\hline p12560 & A_23_P304682 & -0.990915532 & 0.000123416 & up-down & & miRNA sequestration \\
\hline p40979_v4 & A_33_P3391005 & -0.996196971 & 0.000021667 & down-up & & miRNA sequestration \\
\hline p38890_v4 & A_32_P831181 & -0.996877479 & 0.000014610 & up-down & & miRNA sequestration \\
\hline p3356 & A_21_P0014571 & 0.991097745 & 0.000118522 & up-up & Sense & \\
\hline p12621 & A_19_P00317034 & 0.993856369 & 0.000056500 & down-down & Sense & \\
\hline p5975 & A_23_P63660 & -0.991237992 & 0.000114823 & up-down & & miRNA sequestration \\
\hline p9442 & A_23_P63660 & -0.993997587 & 0.000053935 & up-down & & miRNA sequestration \\
\hline p1517 & A_23_P161190 & 0.990268562 & 0.000141591 & up-up & Intergenic $(10 \mathrm{k})$ & \\
\hline p29965 & A_23_P208389 & -0.999649344 & 0.000000184 & down-up & & miRNA sequestration \\
\hline p20045 & A_23_P208389 & -0.993224778 & 0.000068700 & down-up & & miRNA sequestration \\
\hline p33596 & A_23_P146209 & 0.994585549 & 0.000043895 & down-down & & miRNA sequestration \\
\hline p34907_v4 & A_33_P3333554 & 0.992145626 & 0.000092295 & down-down & & miRNA sequestration \\
\hline p38352_v4 & A_23_P155666 & 0.997567735 & 0.000008867 & down-down & & miRNA sequestration \\
\hline p36486_v4 & A_23_P64792 & -0.992286627 & 0.000089015 & down-up & & miRNA sequestration \\
\hline p23949 & A_23_P64792 & 0.992666927 & 0.000080464 & up-up & & miRNA sequestration \\
\hline $\mathrm{p} 25165$ & A_23_P50897 & 0.990700732 & 0.000129312 & down-down & Antisense & \\
\hline p10750 & A_33_P3303305 & 0.991255110 & 0.000114375 & down-down & Intronic & \\
\hline p33919 & A_23_P337201 & 0.997063796 & 0.000012919 & down-down & & miRNA sequestration \\
\hline p1358 & A_21_P0010738 & 0.996673835 & 0.000016577 & down-down & Sense & \\
\hline p6352 & A_23_P163306 & 0.993659613 & 0.000060173 & down-down & & miRNA sequestration \\
\hline p18271 & A_24_P109652 & 0.992664902 & 0.000080508 & up-up & & miRNA sequestration \\
\hline p2107 & A_21_P0006705 & 0.998881367 & 0.000001876 & up-up & Sense & \\
\hline $\mathrm{p} 4653$ & A_23_P304897 & 0.995441532 & 0.000031122 & down-down & Intronic & \\
\hline p4963 & A_23_P304897 & 0.999067618 & 0.000001304 & down-down & Intronic & \\
\hline p39057_v4 & A_33_P3259542 & 0.993876252 & 0.000056136 & up-up & & miRNA sequestration \\
\hline p26034 & A_33_P3259542 & 0.990129120 & 0.000145671 & up-up & & miRNA sequestration \\
\hline p6898 & A_21_P0014351 & 0.993996895 & 0.000053948 & down-down & Sense & \\
\hline p34009_v4 & A_23_P104188 & 0.992257395 & 0.000089690 & down-down & Intergenic (10 k) & \\
\hline p34993_v4 & A_23_P117582 & 0.992864673 & 0.000076188 & up-up & & miRNA sequestration \\
\hline p25051 & A_23_P117582 & 0.990949567 & 0.000122495 & up-up & & miRNA sequestration \\
\hline
\end{tabular}

Dose-response clonogenic survival curves were plotted on a log-linear scale using Graphpad Prism 5 software.

\section{Apoptosis assay}

Apoptotic cells were identified by using the Annexin V-FITC and propidium iodide (PI) apoptosis detection kit (BestBio, Shanghai, China) according to the manufacturer's instructions. Briefly, RS-FaDu and $\mathrm{FaDu}$ cells were seeded at a density of $4 \times 10^{5}$ cells in six-well plates and were incubated for $12 \mathrm{~h}$ before being treated with 4 Gy of radiation. The cells were then harvested at four different time points after X-ray exposure $(0,24,48$, and $72 \mathrm{~h}$, respectively). After being washed twice with PBS, the cells were resuspended in $400 \mu \mathrm{L} 1 \times$ binding buffer and stained with $5 \mu \mathrm{L}$ Annexin V-FITC for $15 \mathrm{~min}$ and $10 \mu \mathrm{L}$ PI for $5 \mathrm{~min}$ at $4^{\circ} \mathrm{C}$ in the dark. Apoptosis was analyzed by a Gallios flow cytometer (Beckman Coulter, Brea, CA, USA). The percentage of total apoptosis was calculated as the sum of the early apoptosis (Annexin 


\begin{tabular}{|c|c|}
\hline Characteristics & No. Patients \\
\hline \multicolumn{2}{|l|}{ Sex } \\
\hline Female & 0 \\
\hline Male & 13 \\
\hline Age (years old) & (Median 57, Range 49-67) \\
\hline \multicolumn{2}{|l|}{ Drinking } \\
\hline Regularly & 9 \\
\hline Occasionally & 4 \\
\hline Seldom & 0 \\
\hline \multicolumn{2}{|l|}{ Smoking } \\
\hline Regularly & 10 \\
\hline Occasionally & 0 \\
\hline Seldom & 3 \\
\hline \multicolumn{2}{|c|}{ *Histological Differentiation } \\
\hline Well-Moderate & 2 \\
\hline Poor & 11 \\
\hline \multicolumn{2}{|l|}{ *Clinical Stage } \\
\hline $\mathrm{I}+\mathrm{II}$ & 1 \\
\hline III + IV & 12 \\
\hline \multicolumn{2}{|l|}{${ }^{*}$ Treatment } \\
\hline $\mathrm{S}+\mathrm{X}$ & 13 \\
\hline
\end{tabular}

*at the first visit.

S: Surgery; X: radiation.

$\mathrm{V}+/ \mathrm{PI}-$ ) and the late apoptosis (Annexin $\mathrm{V}+/ \mathrm{PI}+$ ). The experiments were repeated three times and data were analyzed by Kaluza software (version 1.2; Beckman Coulter).

\section{RNA extraction and microarray analysis}

$\mathrm{RS}-\mathrm{FaDu}$ and $\mathrm{FaDu}$ cells were seeded at a density of $4 \times 10^{5}$ cells in three six-well plates each. They were treated with 4 Gy of radiation and then cultured under the indicated experimental conditions. Cells were harvested at three different time points after X-ray exposure $(0,2$, and $48 \mathrm{~h}$, respectively). Total RNA from each sample was extracted using TRIzol reagent (Invitrogen, Carlsbad, CA, USA) following the manufacturer's instructions. RNA concentration was quantified by the NanoDrop ND-1000 (NanoDrop Technologies/Thermo Scientific, Wilmington, DE, USA), and RNA integrity was assessed by standard denaturing agarose gel electrophoresis. The sample preparation and microarray hybridization were performed based on the manufacturer's standard protocols with minor modifications. Briefly, total RNA was purified after removal of rRNA and tRNA (mRNA-ONLY ${ }^{\mathrm{TM}}$ Eukaryotic mRNA Isolation Kit, Epicentre, Madison, WI, USA). Then, each sample was amplified and transcribed into fluorescent cRNA, along the entire length of the transcript without 3 ' bias, utilizing a random priming method. The labeled cRNAs were hybridized onto the Agilent Human LncRNA v4.0 (4 × 180 K, Arraystar; Agilent Technologies, Santa Clara, CA, USA). The slides were then washed, and the tiff-format original array images were acquired by the Agilent G2505C Scanner (Agilent Technologies).

\section{Microarray data analysis}

The tiff-format original array images were preprocessed via Agilent Feature Extraction software (version 11.0.1.1) and then quantile normalization and differential expression analysis were conducted using the GeneSpring GX software package (version 11.5.1; Agilent Technologies). Cluster analysis and graphical illustration were performed using Cluster 3.0 software. Time-course differentially expressed lncRNAs and mRNAs with statistical significance between the two groups were identified through scatter plot filtering and volcano plot filtering. To filter out outlier samples, we performed hierarchical clustering to show any differences in expression intensity between the clustering group and true group results. The differentially expressed mRNAs were submitted to six pathway databases (KEGG PATHWAY, 
Table 7: Primers used for qRT-PCR

\begin{tabular}{cccc}
\hline mRNAs/lncRNAs & Forward primers $\left(\mathbf{5}^{\prime} \mathbf{-} \mathbf{3}^{\prime}\right)$ & Reverse primers $\left(\mathbf{5}^{\prime} \mathbf{-} \mathbf{3}^{\prime}\right)$ & bp \\
\hline ENST00000470135 & TTGCCAGCAATTCATCAGAG & GGGATATGCCAACCTTGAGA & 151 \\
TCONS_00010875 & TCGTTCACACACCCACTCAT & CGAGTGGGCAAGTTAGTGTG & 153 \\
TCONS_00018436 & CCACCTCAGGATGGAAATGT & TCCCCAACCAAAGTCTTGTC & 160 \\
hox-HOXD10-35 & GCTCCTTCACCACCAACATT & AAATATCCAGGGACGGGAAC & 154 \\
CKMT1A & ACCTGACCCCAGCAGTCTAT & AACACGTTCCACCTCTCGTC & 374 \\
GPNMB & AAGATTGCCACTTGATGCCG & TCCCTCATGTAAGCAGAAGGTC & 75 \\
FBLN5 & CTCACTGTTACCATTCTGGCTC & GACTGGCGATCCAGGTCAAAG & 89 \\
GDA & GCTGGAAGTAGCATAGACCTGC & TCTTCTGCAAAGTCGATGTTCTG & 95 \\
ACTB & GTGGCCGAGGACTTTGATTG & CCTGTAACAACGCATCTCATATT & 73 \\
\hline
\end{tabular}

PID Curated, PID BioCarta, PID Reactome, BioCyc, Reactome, and Panther) for pathway enrichment analysis. After lncRNA and mRNA correlation, lncRNA target prediction included cis-prediction and trans-prediction. To determine cis-prediction, we searched for mRNAs that were in the region of $10 \mathrm{~kb}$ around the lncRNA. Transprediction was based on sequence alignment, which aligns lncRNA to the 3'UTR of mRNA. Then lncRNA-mRNA pairs that share similar sequences were identified from the trans-prediction results. In the lncRNA target prediction analysis, mRNA targets were predicted from cis-prediction and trans-prediction.

\section{Validation of IncRNA and mRNA expression by quantitative real-time polymerase chain reaction}

Quantitative real-time polymerase chain reaction (qRT-PCR) was used to validate the microarray data. Briefly, total RNA was reverse-transcribed to cDNA using SuperScript ${ }^{\mathrm{TM}}$ III Reverse Transcriptase (Invitrogen) following the manufacturer's protocol. qRT-PCR was performed using the SYBR Green chemistry in the GeneAmp PCR System 9700 (ABI Applied Biosystems, Foster City, CA, USA). The forward and reverse primers for validation are listed in Table 7. PCR was performed in a $10-\mu \mathrm{L}$ reaction volume and consisted of an initial denaturation step at $95^{\circ} \mathrm{C}$ for $10 \mathrm{~min}$ followed by amplification with 40 cycles at $95^{\circ} \mathrm{C}$ for $10 \mathrm{sec}$ and $60^{\circ} \mathrm{C}$ for $60 \mathrm{sec}$. The threshold cycle (CT) was defined as the cycle number at which the fluorescence passed a predetermined threshold. Both target and reference ( $\beta$-actin) genes were amplified in separate wells in triplicate. Gene expression was calculated using the comparative threshold cycle $\left(2^{-\Delta C T}\right)$ method.

\section{Lentiviral transfection assay}

Lentivirus containing short hairpin RNA (shRNA) of TCONS 00018436 or empty vectors used as control were purchased from GeneChem (Shanghai, China). And the lentiviral transfection assays were performed following manufacture's instructions. Stably transfected cells were screened by Puromycin $(3 \mathrm{mg} / \mathrm{mL})$ purchased from Sigma.

\section{Statistical analysis}

Data were presented as mean \pm standard deviation (SD) and statistical differences between two experimental groups were determined by using paired $t$-test or Student's $t$-test on SPSS 17.0 software (SPSS Inc., Chicago, IL, USA). Statistical differences in the microarray results were analyzed by fold change (FC) and $P$-value, and the FC and $P$-value were calculated based on normalized data. FC was calculated by computing the ratio of mean intensity of the case group to that of the control group, while $P$-value was calculated using Student's $t$-test. The thresholds for differentially expressed genes were set at FC $\geq 2.0$ and $P$-value $<0.05$. For the lncRNA and mRNA correlation analysis, the Pearson correlation coefficient was calculated to show the correlation between IncRNA and mRNA expression and $P$-value was calculated to show the significance of the Pearson correlation coefficient. Correlation $>0.99$ or correlation $<-0.99$, and $P$-value $<0.05$ were adopted to filter out random relationship. In all analyses, a two-sided $P$-value $<0.05$ was considered statistically significant.

\section{Abbreviations}

HSCC, hypopharyngeal squamous cell carcinoma; CRT, concomitant chemoradiotherapy; IMRT, intensity-modulated radiotherapy; IGRT, image-guided radiotherapy; TOMO, helical tomotherapy; IncRNA, long noncoding RNA; qRT-PCR, quantitative real-time polymerase chain reaction; $\mathrm{GO}$, gene ontology.

\section{ACKNOWLEDGMENTS AND FUNDING}

This work was supported by the Taishan Scholars Program (No. tshw20130950), Shandong Province, and the Department of Science \& Technology of Shandong Province (No. ZR2013HM107, ZR2014HM005, 
2015GSF118014 and 2015GSF118030), the Science Foundation of Qilu Hospital of Shandong University, the Fundamental Research Funds of Shandong University (No.2014QLKY05), and the Zhenjiang Social Development Science \& Technology Funds (SH2014045).

\section{CONFLICTS OF INTEREST}

None.

\section{REFERENCES}

1. Zhou J, Li Y, Wei D, Qian Y, Li W, Liu D, Li G, Pan X, Lei D. Overall survival with and without laryngeal function preservation in 580 patients with hypopharyngeal squamous cell carcinoma. Oncol Rep. 2015; 34:3196-202.

2. Wiezorek T, Brachwitz T, Georg D, Blank E, Fotina I, Habl G, Kretschmer M, Lutters G, Salz H, Schubert K, Wagner D, Wendt TG. Rotational IMRT techniques compared to fixed gantry IMRT and tomotherapy: multiinstitutional planning study for head-and-neck cases. Radiat Oncol. 2011; 6:20.

3. Wycliffe ND, Grover RS, Kim PD, Simental A Jr. Hypopharyngeal cancer. Top Magn Reson Imaging. 2007; 18:243-58.

4. Guo Y, Zhu XD, Qu S, Li L, Su F, Li Y, Huang ST, Li DR. Identification of genes involved in radioresistance of nasopharyngeal carcinoma by integrating gene ontology and protein-protein interaction networks. Int J Oncol. 2012; 40:85-92.

5. Perri F, Pacelli R, Della Vittoria Scarpati G, Cella L, Giuliano M, Caponigro F, Pepe S. Radioresistance in head and neck squamous cell carcinoma: biological bases and therapeutic implications. Head Neck. 2015; 37:763-70.

6. Kapranov P, Willingham AT, Gingeras TR. Genome-wide transcription and the implications for genomic organization. Nat Rev Genet. 2007; 8:413-23.

7. Ernst C, Morton CC. Identification and function of long non-coding RNA. Front Cell Neurosci. 2013; 7:168.

8. Bolton EM, Tuzova AV, Walsh AL, Lynch T, Perry AS. Noncoding RNAs in prostate cancer: the long and the short of it. Clin Cancer Res. 2014; 20:35-43.

9. Jia W, Chen W, Kang J. The functions of microRNAs and long non-coding RNAs in embryonic and induced pluripotent stem cells. Genomics Proteomics Bioinformatics. 2013; 11:275-83.

10. Gupta RA, Shah N, Wang KC, Kim J, Horlings HM, Wong DJ, Tsai MC, Hung T, Argani P, Rinn JL, Wang Y, Brzoska P, Kong B, et al. Long non-coding RNA HOTAIR reprograms chromatin state to promote cancer metastasis. Nature. 2010; 464:1071-76.

11. Cheetham SW, Gruhl F, Mattick JS, Dinger ME. Long noncoding RNAs and the genetics of cancer. Br J Cancer. 2013; 108:2419-25.
12. Wang Q, Fan H, Liu Y, Yin Z, Cai H, Liu J, Wang Z, Shao M, Sun X, Diao J, Liu Y, Tong L, Fan Q. Curcumin enhances the radiosensitivity in nasopharyngeal carcinoma cells involving the reversal of differentially expressed long non-coding RNAs. Int J Oncol. 2014; 44:858-64.

13. Lu H, He Y, Lin L, Qi Z, Ma L, Li L, Su Y. Long noncoding RNA MALAT1 modulates radiosensitivity of HR$\mathrm{HPV}+$ cervical cancer via sponging miR-145. Tumour Biol. 2016; 37:1683-91.

14. Tan J, Qiu K, Li M, Liang Y. Double-negative feedback loop between long non-coding RNA TUG1 and miR-145 promotes epithelial to mesenchymal transition and radioresistance in human bladder cancer cells. FEBS Lett. 2015; 589:3175-81.

15. Skvortsov S, Jimenez CR, Knol JC, Eichberger P, Schiestl B, Debbage P, Skvortsova I, Lukas P. Radioresistant head and neck squamous cell carcinoma cells: intracellular signaling, putative biomarkers for tumor recurrences and possible therapeutic targets. Radiother Oncol. 2011; 101:177-82.

16. Wang WJ, Wu SP, Liu JB, Shi YS, Huang X, Zhang QB, Yao KT. MYC regulation of CHK1 and CHK2 promotes radioresistance in a stem cell-like population of nasopharyngeal carcinoma cells. Cancer Res. 2013; 73:1219-31.

17. Huarte M, Guttman M, Feldser D, Garber M, Koziol MJ, Kenzelmann-Broz D, Khalil AM, Zuk O, Amit I, Rabani M, Attardi LD, Regev A, Lander ES, et al. A large intergenic noncoding RNA induced by p53 mediates global gene repression in the p53 response. Cell. 2010; 142:409-19.

18. Liu Y, Lu X. Non-coding RNAs in DNA damage response. Am J Cancer Res. 2012; 2:658-75.

19. Liu Y, Qian J, Li X, Chen W, Xu A, Zhao K, Hua Y, Huang Z, Zhang J, Liang C, Su S, Li P, Shao P, et al. Long noncoding RNA BX357664 regulates cell proliferation and epithelial-to-mesenchymal transition via inhibition of TGF- $\beta 1 / \mathrm{p} 38 / \mathrm{HSP} 27$ signaling in renal cell carcinoma. Oncotarget. 2016; 7:81410-22. doi: 10.18632/ oncotarget. 12937.

20. Sun M, Nie F, Wang Y, Zhang Z, Hou J, He D, Xie M, Xu L, De W, Wang Z, Wang J. LncRNA HOXA11-AS Promotes Proliferation and Invasion of Gastric Cancer by Scaffolding the Chromatin Modification Factors PRC2, LSD1, and DNMT1. Cancer Res. 2016; 76:6299-310.

21. Li X, Wang H, Yao B, Xu W, Chen J, Zhou X. lncRNA H19/miR-675 axis regulates cardiomyocyte apoptosis by targeting VDAC1 in diabetic cardiomyopathy. Sci Rep. 2016; 6:36340.

22. Ren K, Li Y, Lu H, Li Z, Li Z, Wu K, Li Z, Han X. Long Noncoding RNA HOTAIR Controls Cell Cycle by Functioning as a Competing Endogenous RNA in Esophageal Squamous Cell Carcinoma. Transl Oncol. 2016; 9:489-97.

23. Chang L, Graham P, Hao J, Ni J, Deng J, Bucci J, Malouf D, Gillatt D, Li Y. Cancer stem cells and signaling pathways in radioresistance. Oncotarget. 2016; 7:11002-17. doi: 10.18632/oncotarget.6760. 
24. Jin C, Yan B, Lu Q, Lin Y, Ma L. The role of MALAT1/ $\mathrm{miR}-1 / \mathrm{slug}$ axis on radioresistance in nasopharyngeal carcinoma. Tumour Biol. 2016; 37:4025-33.

25. Lu Y, Li T, Wei G, Liu L, Chen Q, Xu L, Zhang K, Zeng D, Liao R. The long non-coding RNA NEAT1 regulates epithelial to mesenchymal transition and radioresistance in through miR-204/ZEB1 axis in nasopharyngeal carcinoma. Tumour Biol. 2016; 37:11733-41.

26. Zhang H, Luo H, Hu Z, Peng J, Jiang Z, Song T, Wu B, Yue J, Zhou R, Xie R, Chen T, Wu S. Targeting WISP1 to sensitize esophageal squamous cell carcinoma to irradiation. Oncotarget. 2015; 6:6218-34. doi: 10.18632/ oncotarget.3358.

27. Borràs-Fresneda $\mathrm{M}$, Barquinero JF, Gomolka $\mathrm{M}$, Hornhardt S, Rössler U, Armengol G, Barrios L. Differences in DNA Repair Capacity, Cell Death and Transcriptional Response after Irradiation between a Radiosensitive and a Radioresistant Cell Line. Sci Rep. 2016; 6:27043.

28. Michna A, Schötz U, Selmansberger M, Zitzelsberger H, Lauber K, Unger K, Hess J. Transcriptomic analyses of the radiation response in head and neck squamous cell carcinoma subclones with different radiation sensitivity: time-course gene expression profiles and gene association networks. Radiat Oncol. 2016; 11:94.

29. Li G, Liu Y, Liu C, Su Z, Ren S, Wang Y, Deng T, Huang D, Tian Y, Qiu Y. Genome-wide analyses of long noncoding RNA expression profiles correlated with radioresistance in nasopharyngeal carcinoma via next-generation deep sequencing. BMC Cancer. 2016; 16:719.

30. Yogev O, Barker K, Sikka A, Almeida GS, Hallsworth A, Smith LM, Jamin Y, Ruddle R, Koers A, Webber HT, Raynaud FI, Popov S, Jones C, et al. p53 Loss in MYCDriven Neuroblastoma Leads to Metabolic Adaptations Supporting Radioresistance. Cancer Res. 2016; 76:3025-35.

31. Zheng R, Yao Q, Xie G, Du S, Ren C, Wang Y, Yuan Y. TAT-ODD-p53 enhances the radiosensitivity of hypoxic breast cancer cells by inhibiting Parkin-mediated mitophagy. Oncotarget. 2015; 6:17417-29. doi: 10.18632/oncotarget.4002.

32. Jun S, Jung YS, Suh HN, Wang W, Kim MJ, Oh YS, Lien EM, Shen X, Matsumoto Y, McCrea PD, Li L, Chen J, Park JI. LIG4 mediates Wnt signalling-induced radioresistance. Nat Commun. 2016; 7:10994.

33. Engreitz JM, Haines JE, Perez EM, Munson G, Chen J, Kane M, McDonel PE, Guttman M, Lander ES. Local regulation of gene expression by 1 ncRNA promoters, transcription and splicing. Nature. 2016; 539:452-55.

34. Ørom UA, Derrien T, Beringer M, Gumireddy K, Gardini A, Bussotti G, Lai F, Zytnicki M, Notredame C, Huang Q, Guigo R, Shiekhattar R. Long noncoding RNAs with enhancer-like function in human cells. Cell. 2010; 143:46-58.

35. Hung T, Wang Y, Lin MF, Koegel AK, Kotake Y, Grant GD, Horlings HM, Shah N, Umbricht C, Wang P, Wang Y, Kong B, Langerød A, et al. Extensive and coordinated transcription of noncoding RNAs within cell-cycle promoters. Nat Genet. 2011; 43:621-29.
36. Schmitt AM, Chang HY. Long Noncoding RNAs in Cancer Pathways. Cancer Cell. 2016; 29:452-63.

37. Shao Y, Ye M, Li Q, Sun W, Ye G, Zhang X, Yang Y, Xiao B, Guo J. LncRNA-RMRP promotes carcinogenesis by acting as a miR-206 sponge and is used as a novel biomarker for gastric cancer. Oncotarget. 2016; 7:3781224. doi: 10.18632/oncotarget.9336.

38. Wang ZH, Guo XQ, Zhang QS, Zhang JL, Duan YL, Li GF, Zheng DL. Long non-coding RNA CCAT1 promotes glioma cell proliferation via inhibiting microRNA-410. Biochem Biophys Res Commun. 2016; 480:715-20.

39. Zhou Q, Chen F, Zhao J, Li B, Liang Y, Pan W, Zhang S, Wang X, Zheng D. Long non-coding RNA PVT1 promotes osteosarcoma development by acting as a molecular sponge to regulate miR-195. Oncotarget. 2016; 7:82620-33. doi: 10.18632/oncotarget.13012.

40. Xie CH, Cao YM, Huang Y, Shi QW, Guo JH, Fan ZW, Li JG, Chen BW, Wu BY. Long non-coding RNA TUG1 contributes to tumorigenesis of human osteosarcoma by sponging miR-9-5p and regulating POU2F1 expression. Tumour Biol. 2016; 37:15031-41.

41. Song L, Liu S, Zhang L, Yao H, Gao F, Xu D, Li Q. MiR-21 modulates radiosensitivity of cervical cancer through inhibiting autophagy via the PTEN/Akt/HIF-1 $\alpha$ feedback loop and the AktmTOR signaling pathway. Tumour Biol. 2016; 37:12161-68.

42. Qu JQ, Yi HM, Ye X, Li LN, Zhu JF, Xiao T, Yuan L, Li JY, Wang YY, Feng J, He QY, Lu SS, Yi H, Xiao ZQ. MiR23a sensitizes nasopharyngeal carcinoma to irradiation by targeting IL-8/Stat3 pathway. Oncotarget. 2015; 6:28341-56. doi: 10.18632/oncotarget.5117.

43. Qu JQ, Yi HM, Ye X, Zhu JF, Yi H, Li LN, Xiao T, Yuan L, Li JY, Wang YY, Feng J, He QY, Lu SS, Xiao ZQ. MiRNA-203 Reduces Nasopharyngeal Carcinoma Radioresistance by Targeting IL8/AKT Signaling. Mol Cancer Ther. 2015; 14:2653-64.

44. Sun Q, Liu T, Zhang T, Du S, Xie GX, Lin X, Chen L, Yuan Y. MiR-101 sensitizes human nasopharyngeal carcinoma cells to radiation by targeting stathmin 1 . Mol Med Rep. 2015; 11:3330-36.

45. Yang H, Liu P, Zhang J, Peng X, Lu Z, Yu S, Meng Y, Tong WM, Chen J. Long noncoding RNA MIR31HG exhibits oncogenic property in pancreatic ductal adenocarcinoma and is negatively regulated by miR-193b. Oncogene. 2016; 35:3647-57.

46. Duan W, Du L, Jiang X, Wang R, Yan S, Xie Y, Yan K, Wang Q, Wang L, Zhang X, Pan H, Yang Y, Wang C. Identification of a serum circulating lncRNA panel for the diagnosis and recurrence prediction of bladder cancer. Oncotarget. 2016; 7:78850-58. doi: 10.18632/oncotarget.12880.

47. Zhang K, Luo Z, Zhang Y, Zhang L, Wu L, Liu L, Yang J, Song X, Liu J. Circulating lncRNA H19 in plasma as a novel biomarker for breast cancer. Cancer Biomark. 2016; 17:187-94.

48. Jiang C, Li X, Zhao H, Liu H. Long non-coding RNAs: potential new biomarkers for predicting tumor invasion and metastasis. Mol Cancer. 2016; 15:62. 